\title{
Akap1 deficiency exacerbates diabetic cardiomyopathy in mice by NDUFS1-mediated mitochondrial dysfunction and apoptosis
}

\author{
Bingchao $\mathrm{Qi}^{1} \cdot$ Linjie $\mathrm{He}^{2,3} \cdot \mathrm{Ya} \mathrm{Zhao}^{2,3,4} \cdot$ Ling Zhang ${ }^{5} \cdot$ Yuanfang $\mathrm{He}^{2,3} \cdot$ Jun $\mathrm{Li}^{2,3} \cdot$ Congye $\mathrm{Li}^{5} \cdot$ Bo Zhang ${ }^{2,3}$. \\ Qichao Huang ${ }^{2,3} \cdot$ Jinliang Xing ${ }^{2,3} \cdot \mathrm{Fei}^{\mathrm{Li}^{5}} \cdot \mathrm{Yan} \mathrm{Li}^{1} \cdot \mathrm{Lele} \mathrm{Ji}^{6}$ (D)
}

Received: 13 November 2019 / Accepted: 6 January 2020 / Published online: 19 February 2020

(C) Springer-Verlag GmbH Germany, part of Springer Nature 2020

\begin{abstract}
Aims/hypothesis Diabetic cardiomyopathy, characterised by increased oxidative damage and mitochondrial dysfunction, contributes to the increased risk of heart failure in individuals with diabetes. Considering that A-kinase anchoring protein 121 (AKAP1) is localised in the mitochondrial outer membrane and plays key roles in the regulation of mitochondrial function, this study aimed to investigate the role of AKAP1 in diabetic cardiomyopathy and explore its underlying mechanisms.

Methods Loss- and gain-of-function approaches were used to investigate the role of AKAP1 in diabetic cardiomyopathy. Streptozotocin (STZ) was injected into Akapl-knockout (Akapl-KO) mice and their wild-type (WT) littermates to induce diabetes. In addition, primary neonatal cardiomyocytes treated with high glucose were used as a cell model of diabetes. Cardiac function was assessed with echocardiography. Akapl overexpression was conducted by injecting adeno-associated virus 9 carrying Akap1 (AAV9-Akap1). LC-MS/MS analysis and functional experiments were used to explore underlying molecular mechanisms.

Results AKAP1 was downregulated in the hearts of STZ-induced diabetic mouse models. Akap1-KO significantly aggravated cardiac dysfunction in the STZ-treated diabetic mice when compared with WT diabetic littermates, as evidenced by the left ventricular ejection fraction (LVEF; STZ-treated WT mice [WT/STZ] vs STZ-treated Akap1-KO mice [KO/STZ], 51.6\% vs 41.6\%). Mechanistically, Akapl deficiency impaired mitochondrial respiratory function characterised by reduced ATP production. Additionally, Akapl deficiency increased cardiomyocyte apoptosis via enhanced mitochondrial reactive oxygen species (ROS) production. Furthermore, immunoprecipitation and mass spectrometry analysis indicated that AKAP1 interacted with the NADH-ubiquinone oxidoreductase $75 \mathrm{kDa}$ subunit (NDUFS1). Specifically, Akapl deficiency inhibited complex I activity by preventing translocation of NDUFS1 from the cytosol to mitochondria. Akap1 deficiency was also related to decreased ATP production and enhanced mitochondrial ROS-related apoptosis. In contrast, restoration of AKAP1 expression in the hearts of STZ-treated diabetic mice promoted translocation of NDUFS1 to mitochondria and alleviated diabetic cardiomyopathy in the
\end{abstract}

Bingchao Qi, Linjie He and Ya Zhao contributed equally to this work.

Electronic supplementary material The online version of this article (https://doi.org/10.1007/s00125-020-05103-w) contains peer-reviewed but unedited supplementary material, which is available to authorised users.

\section{Lele Ji}

jilele@fmmu.edu.cn

$\triangle$ Yan $\mathrm{Li}$

profleeyan@163.com

$\triangle \mathrm{Fei} \mathrm{Li}$

lifei2288@hotmail.com

1 Department of Cardiology, Tangdu Hospital, Fourth Military Medical University, 1 Xinsi Road, Xi'an 710038, China

2 State Key Laboratory of Cancer Biology, Fourth Military Medical University, Xi'an, China
3 Department of Physiology and Pathophysiology, Fourth Military Medical University, Xi'an, China

4 Laboratory Animal Center, Fourth Military Medical University, Xi'an, China

5 Department of Cardiology, Xijing Hospital, Fourth Military Medical University, 169 Changle West Road, Xi'an 710032, China

6 Experimental Teaching Center of Basic Medicine, Fourth Military Medical University, 169 Changle West Road, Xi'an 710032, China 


\section{Research in context}

\section{What is already known about this subject?}

- Diabetic cardiomyopathy is characterised by mitochondrial dysfunction

- A-kinase anchoring protein 121 (AKAP1) plays an important role in the regulation of mitochondrial function

- $\quad \mathrm{NADH}$-ubiquinone oxidoreductase $75 \mathrm{kDa}$ subunit (NDUFS1) is essential for maintaining mitochondrial complex I activity

\section{What is the key question?}

- What are the roles and molecular mechanisms of AKAP1 in the pathogenesis of diabetic cardiomyopathy?

\section{What are the new findings?}

- $\quad$ AKAP1 is downregulated in streptozotocin-induced diabetic mouse models

- $\quad$ AKAP1 binds with NDUFS1 and promotes its location into mitochondria to regulate complex I activity

- Akap1 deficiency exacerbates diabetic cardiomyopathy by NDUFS1-mediated mitochondrial dysfunction and apoptosis

How might this impact on clinical practice in the foreseeable future?

- Targeting AKAP1 provides the possibility of a new therapeutic approach for diabetic cardiomyopathy and related cardiac diseases

LVEF (WT/STZ injected with adeno-associated virus carrying $g f p$ [AAV9-gfp] vs WT/STZ AAV9-Akap1, 52.4\% vs 59.6\%; KO/ STZ AAV9-gfp vs KO/STZ AAV9-Akap1, $42.2 \%$ vs $57.6 \%$ ).

Conclusions/interpretation Our study provides the first evidence that Akapl deficiency exacerbates diabetic cardiomyopathy by impeding mitochondrial translocation of NDUFS1 to induce mitochondrial dysfunction and cardiomyocyte apoptosis. Our findings suggest that Akapl upregulation has therapeutic potential for myocardial injury in individuals with diabetes.

Keywords AKAP1 · Apoptosis · Diabetic cardiomyopathy · Mitochondrial dysfunction · NDUFS1

Abbreviations

AAV Adeno-associated virus

AAV9-Akapl Adeno-associated virus carrying Akapl

AAV9-gfp Adeno-associated virus carrying $g f p$

Ad-Akap 1

Ad-EV

Ad- $g f p$

AKAP1

Akap1-KO

Co-IP

E/A ratio

\section{IVSd}

$\mathrm{KO} / \mathrm{STZ}$

LVEF

LVFS

LVIDd

LVIDs

NDUFS1

A-kinase anchoring protein 121
Adenoviral vectors carrying Akapl

Empty adenoviral vectors

Adenoviral vectors carrying $g f p$

Akap1-knockout (mice)

Co-immunoprecipitation

Early mitral diastolic wave/late

mitral diastolic wave ratio

Interventricular septum at diastole

STZ-treated Akapl-KO mice

Left ventricular ejection fraction

Left ventricular fractional shorting

Left ventricular diastolic internal dimension

Left ventricular systolic internal dimension

NADH-ubiquinone oxidoreductase

$75 \mathrm{kDa}$ subunit

$\begin{array}{ll}\text { OCR } & \text { Oxygen consumption rate } \\ \text { OXPHOS } & \text { Oxidative phosphorylation } \\ \text { qPCR } & \text { Quantitative real-time PCR } \\ \text { ROS } & \text { Reactive oxygen species } \\ \text { SIAH2 } & \text { Siah E3 ubiquitin protein ligase 2 } \\ \text { siAkap1 } & \text { Akap1-specific small interfering RNA } \\ \text { STZ } & \text { Streptozotocin } \\ \text { TEM } & \text { Transmission electron microscopy } \\ \text { WT } & \text { Wild-type } \\ \text { WT/STZ } & \text { STZ-treated WT mice }\end{array}$

\section{Introduction}

The number of adults with diabetes in the world has increased from 108 million in 1980 to 422 million in 2014, and these figures are expected to increase to 693 million by $2045[1,2]$. Cardiovascular disease is the leading cause of mortality in individuals with diabetes, regardless of whether it is type 1 or type 2 
[3]. Diabetic cardiomyopathy is a pathophysiological condition in which heart failure occurs independent of artery disease, hypertension and valvular heart disease [4]. It is relatively clear that diabetic cardiomyopathy is characterised by abnormal cardiac systolic and diastolic function; however, the specific pathological mechanism of diabetic cardiomyopathy has not been completely elucidated and existing therapies are limited.

The working heart is a high energy-demanding tissue and mitochondria are the primary energy-generating organelles in the heart. The vast majority of ATP is produced through mitochondrial oxidative phosphorylation (OXPHOS). Acting as primary ATP suppliers and the main source of reactive oxygen species (ROS) in cardiomyocytes, mitochondria are intimately involved in the pathogenesis of diabetic cardiomyopathy. On the one hand, ATP production is significantly reduced in diabetic cardiomyopathy $[5,6]$. On the other hand, destruction of the oxidative-respiratory chain is accompanied by increased ROS production and cardiomyocyte apoptosis [7, 8]. Accumulating evidence has indicated that diabetic cardiomyopathy involves mitochondrial dysfunction and increased mitochondrial ROS production [5, 9]. However, the causes of mitochondrial dysfunction and enhanced mitochondrial ROS production in diabetic cardiomyopathy remain incompletely understood.

A-kinase anchoring protein 121 (AKAP1) anchors protein kinase $\mathrm{A}$ to the mitochondrial outer membrane. Emerging evidence has shown that AKAP1 brings various molecules from the cytosol to mitochondria and that it regulates factors associated with mitochondrial physiological activities, including mitochondrial $\mathrm{Ca}^{2+}$, mitochondrial membrane potential and mitochondrial dynamics [10-13]. Mitochondria are the major energy source for cardiac contraction and relaxation. Mitochondrial dysfunction and ROS-mediated apoptosis are critical factors in the pathogenesis of diabetic cardiomyopathy. A previous study has reported that Akap1 downregulation aggravates mitochondrial dysfunction and increases oxidative stress under a pressure overload model in rats [14]. However, the function of AKAP1 in diabetic cardiomyopathy needs to be explored.

In this study, we investigated the role of AKAP1 in diabetic cardiomyopathy and elucidated the underlying molecular mechanisms.

\section{Methods}

Animals All of the animal experiments were conducted in accordance with the Guide for the Care and Use of Laboratory Animals, eighth edition (2011) and the study was approved by the Fourth Military Medical University Committee on Animal Care. Akapl-knockout (Akapl-KO) mice (C57BL/6J background) were kindly provided by J. Xing (Department of Physiology and Pathophysiology, The Fourth Military Medical University, Xi'an, China). All of the mice were maintained under identical conditions of temperature $(22 \pm$ $\left.0.5^{\circ} \mathrm{C}\right)$, humidity $(60 \pm 5 \%)$ and $12 \mathrm{~h}$ light-dark cycle, and they drank and ate freely. For this study, measurements were taken from distinct samples/animals. Animals were randomly assigned and the experimenters were blind to histology assays, tests of in vivo function and outcome assessment.

Experimental diabetic model Diabetes was induced in 8-weekold male mice by consecutive intraperitoneal injections of streptozotocin (STZ; Sigma-Aldrich, St. Louis, MO, USA) $50 \mathrm{mg} / \mathrm{kg}$ dissolved in $0.1 \mathrm{~mol} / \mathrm{l}$ citrate buffer, $\mathrm{pH} 4.5$ ) for 5 days. Blood glucose was measured using mouse tail vein blood on the seventh day after the last STZ injection. Mice were considered to be diabetic and enrolled in the present study only if they had hyperglycaemia (>16.7 mmol/l). Control mice were intraperitoneally injected with the same amount of citrate buffer (vehicle). All of the mice continued to be fed for 12 weeks, and blood glucose and body weight were regularly checked. Mouse hearts were isolated at 0,6 and 12 weeks after STZ injection, for further analysis.

Neonatal rat cardiomyocyte culture Primary neonatal cardiomyocytes were isolated from neonatal rats $(0-2$ days old), as previously described [5]. The primary neonatal cardiomyocytes were subjected to normal-glucose medium (5.5 mmol/l glucose) and high-glucose medium $(30 \mathrm{mmol} / \mathrm{l}$ glucose) for $48 \mathrm{~h}$.

Western blotting Cardiac tissues and cultured cardiomyocytes were collected and homogenised with RIPA buffer (1\% Triton $\mathrm{X}-100,1 \%$ deoxycholate, $0.1 \% \mathrm{SDS}, \mathrm{wt} / \mathrm{vol}$.). The antibodies used and the dilution ratios are shown in electronic supplementary material (ESM) Table 1. See ESM Methods for details.

Quantitative real-time PCR Quantitative real-time PCR (qPCR) was performed with SYBR Premix Ex Taq II (Takara, Otsu, Japan). The primer sequences are shown in ESM Table 2. See ESM Methods for details.

Histological analysis The cardiac tissues of the mice were fixed overnight in 4\% (wt/vol.) paraformaldehyde, embedded in paraffin and continuously cut into $5 \mathrm{~mm}$-thick slices. Masson staining was performed with a standard procedure to assess cardiac collagen content, as described previously [15].

Immunohistochemistry and immunofluorescence analysis Immunohistochemical staining of cardiac sections and immunofluorescence analysis of primary neonatal cardiomyocytes were performed as described previously [15]. The antibodies used and the dilution ratios are shown in ESM Table 1.

MitoTracker staining To view mitochondrial location, primary neonatal cardiomyocytes were stained with $100 \mathrm{~mol} / 1$ MitoTracker Red CMXRos probe (Thermo Fisher Scientific, 
Waltham, MA, USA) at $37^{\circ} \mathrm{C}$ for $30 \mathrm{~min}$. Images were obtained with a confocal laser scanning microscope (Nikon A1R $\mathrm{MP}+$ Confocal Microscope; Nikon, Tokyo, Japan).

Echocardiography Echocardiography was performed in Mmode with a Vevo 2100 echocardiography system (VisualSonics, Toronto, ON, Canada) to record the left ventricular systolic and diastolic motion profile, as described previously [5]. See ESM Methods for details.

Haemodynamic measurements In vivo cardiac haemodynamic function was evaluated with a Millar tip-pressure catheter (Millar Instruments, Houston, TX, USA), as previously described [16]. See ESM Methods for details.

Apoptosis assay TUNEL staining was performed for analysis of heart tissue apoptosis in a standard procedure using a terminal deoxynucleotidyl TUNEL assay kit (SigmaAldrich). Staining with DAPI and TUNEL was performed to confirm apoptotic nuclei. A mean of ten random fields was analysed per animal. For analysis of primary neonatal cardiomyocytes, apoptosis assay was performed using the Annexin V-FITC Apoptosis Detection Kit (Beyotime, Shanghai, China). Cardiomyocytes were double stained with Annexin V-FITC and propidium iodide (PI) according to the manufacturer's protocol. Analysis of flow cytometry (COULTER EPICS XL-MCL; Beckman Coulter, Brea, CA, USA) was performed as previously described [17]. See ESM Methods for details.

Transmission electron microscopy Mitochondrial morphology in the left ventricle of the heart was detected with transmission electron microscopy (TEM). See ESM Methods for details.

Detection of mitochondrial ROS Mitochondrial ROS were detected using the fluorescent probe MitoSOX (Invitrogen, Waltham, MA, USA), following the manufacturer's protocols. Images were obtained with a confocal laser scanning microscope and analysed with ImageJ software (Rawak Software, Stuttgart, Germany).

Mitochondrial membrane potential Primary neonatal cardiomyocytes were co-incubated with JC-1 (Invitrogen), then detected using flow cytometry, as previously described [5]. See ESM Methods for details.

Mitochondrial oxygen consumption rate Mitochondrial oxygen consumption rate (OCR) of primary neonatal cardiomyocytes treated with small interfering RNA or adenoviruses (see below for details) was analysed with a XF24 Extracellular Flux Analyzer (Agilent Seahorse Bioscience, Santa Clara, CA, USA), as previously described [18]. Oligomycin, trifluoromethoxy carbonyl cyanide phenylhydrazone (FCCP), rotenone and antimycin A were used as inhibitors. See ESM Methods for details.

Upregulation and downregulation of target genes For animal experiments, adeno-associated virus 9 carrying Akap 1 (AAV9-Akap1) and the control virus were constructed by GeneChem Technology (Shanghai, China). Upregulation of Akapl was performed by intramyocardial injection of AAV9-Akap1. For cell experiments, adenoviral vectors carrying Akapl (Ad-Akapl) and the control viruses (empty adenoviral vectors [Ad-EV] or adenoviral vectors carrying $g f p$ [Ad$g f p]$ ) were constructed by Hanbio Technology (Shanghai, China). Upregulation of the target genes was performed by infection of Ad-Akap1. Small interfering RNAs were designed and synthesised by GenePharma Company (Shanghai, China). Downregulation of target genes was performed by transfection of cardiomyocytes with Akaplspecific small interfering RNA (siAkapl) or Ndufs1-specific

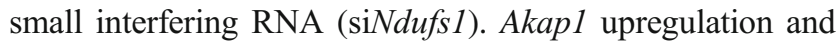
downregulation experiments in cardiomyocytes were conducted under normal-glucose $(5.5 \mathrm{mmol} / \mathrm{l})$ or highglucose $(30 \mathrm{mmol} / \mathrm{l})$ conditions. See ESM Methods for details.

Co-immunoprecipitation Co-immunoprecipitation (Co-IP) was performed on heart samples using a Pierce Classic Magnetic IP/Co-IP Kit (Thermo Fisher Scientific) according to the manufacturer's protocol. See ESM Methods for details.

LC-MS/MS analysis for AKAP1-interacting proteins To identify AKAP1-interacting proteins, the co-immunoprecipitated proteins were visualised after separation by electrophoresis using a Fast Silver Stain Kit (Beyotime) following the manufacturer's protocol. The differential silver-stained gel spots were excised and sent to Novogene Genetics (Beijing, China) for LC-MS/MS analysis. See ESM Methods for details.

Isolation of mitochondria Mitochondria were isolated from mouse hearts using the Tissue Mitochondria Isolation Kit (Beyotime), according to the manufacturer's instructions.

Mitochondrial complex I activity assay Mitochondrial complex I activity was measured using a Mitochondrial Complex I Activity Assay Kit (Solarbio, Beijing, China) following the manufacturer's instructions.

Statistical analysis Data are expressed as mean \pm SD. An unpaired Student's $t$ test was performed for analysis of differences between two groups. For multiple groups, one-way ANOVA was carried out followed by the Bonferroni post hoc test. Statistical analyses were performed with GraphPad Prism 6.0 (GraphPad Software, La Jolla, CA, USA). A value of $p<0.05$ was considered significant. 


\section{Results}

AKAP1 expression is decreased in hearts of diabetic mice To assess the functional role of AKAP1 in the heart in diabetes, we first examined the expression level of AKAP1 in the heart tissues of STZ-induced diabetic mouse models. Compared with control hearts, AKAP1 expression was significantly decreased in the heart of mice 6 weeks after STZ injection (Fig. 1a). The downregulation of AKAP1 was even more obvious in hearts 12 weeks after STZ injection. Similarly, qPCR showed that the mRNA level of Akapl was also significantly decreased in hearts both 6 and 12 weeks after STZ injection (Fig. 1b). Immunohistochemical staining analysis also indicated that AKAP1 expression was decreased in hearts of diabetic mice when compared with wild-type (WT) mice (Fig. 1c). Given the decrease in both protein levels and mRNA levels of AKAP1, the involvement of AKAP1 in diabetic cardiomyopathy is strongly suggested.

\section{Akap1 deficiency exacerbates diabetes-induced cardiac} dysfunction Akap1-KO mice were used to investigate a direct relationship between Akapl deficiency and diabetic cardiomyopathy. The knockout of Akapl in heart tissues was detected by western blotting and immunohistochemical staining (Fig. $2 \mathrm{a}, \mathrm{b})$. The blood glucose level and body weight of STZinduced diabetic mice exhibited a very consistent change with that previously reported (Fig. 2c; ESM Fig. 1a) [19, 20]. Echocardiography analysis showed that knockout and WT mice in the vehicle groups exhibited similar measurements of cardiac function (Fig. 2d-h). However, when compared with STZ-treated WT mice (WT/STZ), STZ-treated Akapl$\mathrm{KO}$ mice (KO/STZ) had notably decreased cardiac contractile function, which was exemplified by decreased left ventricular ejection fraction (LVEF; WT/STZ vs KO/STZ, $51.6 \%$ vs $41.6 \%$ ), and other parameters including decreased left ventricular fractional shorting (LVFS), increased left ventricular systolic internal dimension (LVIDs) and left ventricular diastolic internal dimension (LVIDd) (Fig. 2d-h). Decreased interventricular septum at systole (IVSs) and interventricular septum at diastole (IVSd) were also detected in $\mathrm{KO} / \mathrm{STZ}$ mice vs WT/STZ, however the change in IVSd was not significant (ESM Fig. 1b, c). Furthermore, mitral flow pattern analysis by pulsed Doppler indicated that Akapl knockout exacerbated diastolic dysfunction in diabetic mice, as demonstrated by a reduced early mitral diastolic wave/late mitral diastolic wave ratio (E/A ratio) when compared with control mice (Fig. 2i, j). Additionally, haemodynamic analysis by the Millar transducer showed a decreased peak derivative of pressure over time $(\mathrm{dP} /$ $\mathrm{dt}_{\text {max }}$; a measure of cardiac contractility) and increased minimum peak derivative of pressure over time $\left(\mathrm{dP} / \mathrm{dt}_{\mathrm{min}}\right.$; a measure of cardiac relaxation) in $\mathrm{KO} / \mathrm{STZ}$ mice when compared with WT/STZ mice (Fig. 21, m). Similarly, there were significant decreases in the left ventricular end-systolic pressure (LVESP) and increases in the left ventricular enddiastolic pressure (LVEDP) in KO/STZ vs WT/STZ (ESM Fig. 1d, e). As a pathologically characteristic change, myocardial fibrosis in diabetic cardiomyopathy has been reported in several studies $[19,21]$. Consistently, our data showed that the hearts of diabetic mice exhibited a significant increase in collagen fibres in the interstitial space vs vehicle-treated animals, suggesting the occurrence of myocardial fibrosis (ESM Fig. 2a). In addition, collagen deposition was further increased in the hearts of $\mathrm{KO} / \mathrm{STZ}$ when compared with WT/ STZ (ESM Fig. 2a). As additional evidence, KO/STZ exhibited significant increases in mRNA abundance of common fibrotic markers vs WT/STZ (ESM Fig. 2b). Baseline
Fig. 1 AKAP1 expression is decreased in hearts of mouse models of diabetes. (a) Western blotting and associated quantitative analysis of heart AKAP1 protein expression at 0,6 and 12 weeks after STZ injection; $n=4$ mice. (b) qPCR analysis of heart Akap1 mRNA expression at 0,6 and 12 weeks after STZ injection; $n=4$ mice. (c)

Representative

immunohistochemical staining of AKAP1 (brown) in mouse hearts at 0,6 and 12 weeks after STZ injection. Haematoxylin-stained nuclei are shown in blue. Scale bar, $50 \mu \mathrm{m}$. Data are shown as mean \pm SD. $* p<0.05, * * p<0.01$
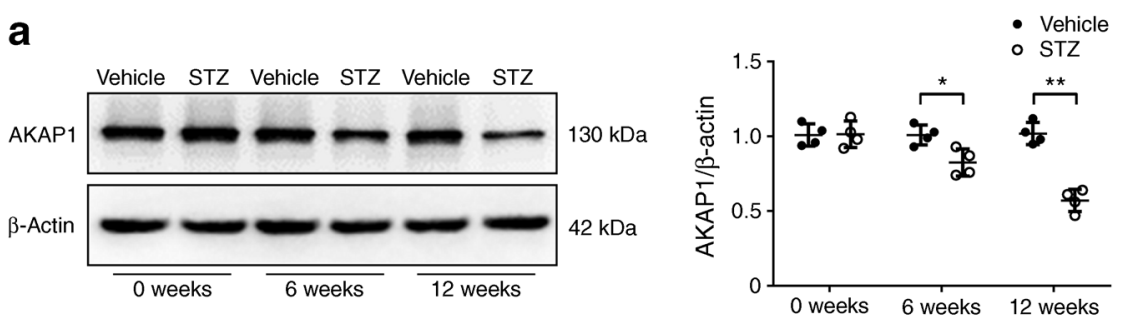

b

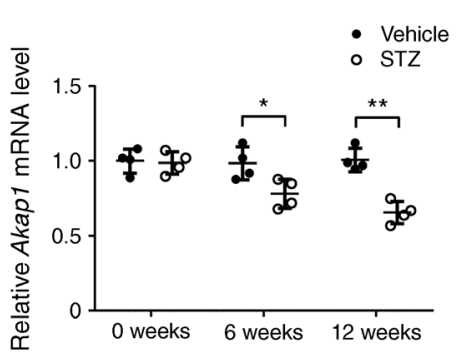

C

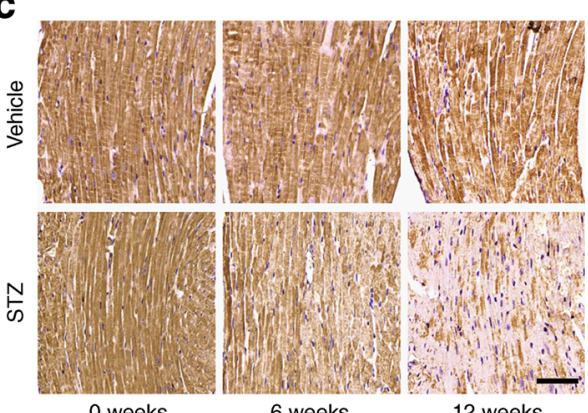


Fig. 2 Akap1 deficiency exacerbates diabetes-induced cardiac dysfunction. (a) Representative western blot of AKAP1 in WT mice and knockout (KO) mice; $n=3$ mice. (b) Representative immunohistochemical staining of AKAP1 in WT mice and KO mice. (c) Blood glucose levels in the different groups; $n=10$ mice. (d) Representative echocardiography images; LVIDd and LVIDs are labelled. (e-h) Echocardiography data analysis; $n=8$ mice. (i, j) Representative Doppler echocardiography images and relative quantification of the E/A ratio; $n=8$ mice. (km) Haemodynamic analysis. Peak derivative of pressure over time $\left(\mathrm{dP} / \mathrm{dt}_{\max }\right)$ is the maximal value of the instantaneous first derivative of left ventricular pressure; minimum peak derivative of pressure over time $\left(\mathrm{dP} / \mathrm{dt}_{\min }\right)$ is the minimum value of the instantaneous first derivative of left ventricular pressure; $n=5$ mice. Data are shown as mean \pm SD. $* p<0.05$, $* * p<0.01$

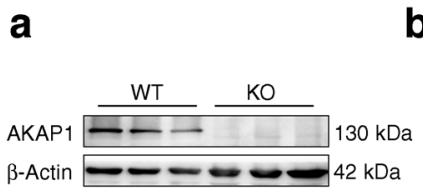

b

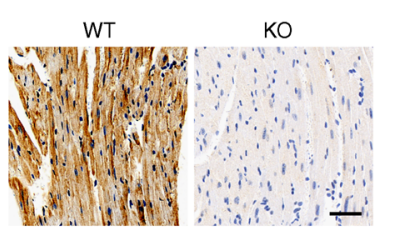

d
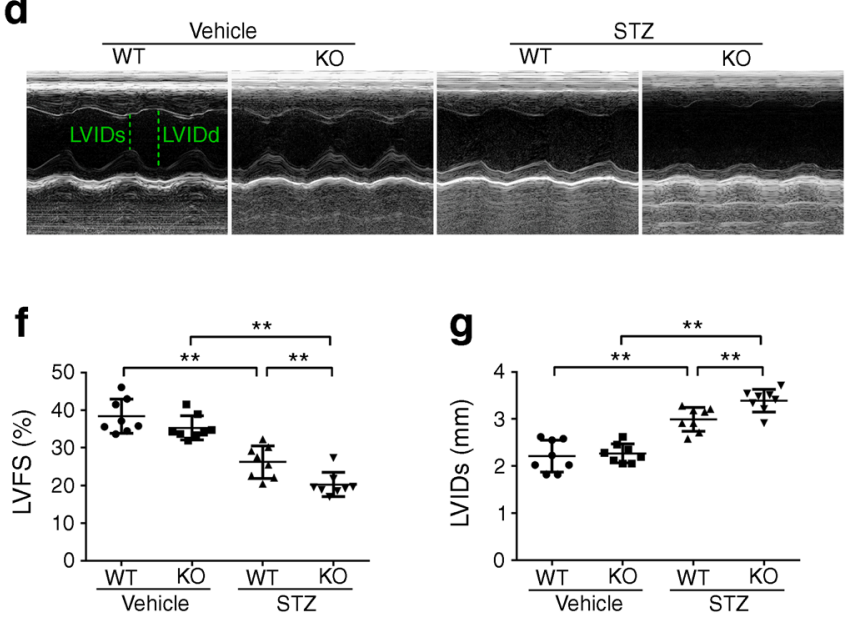

i
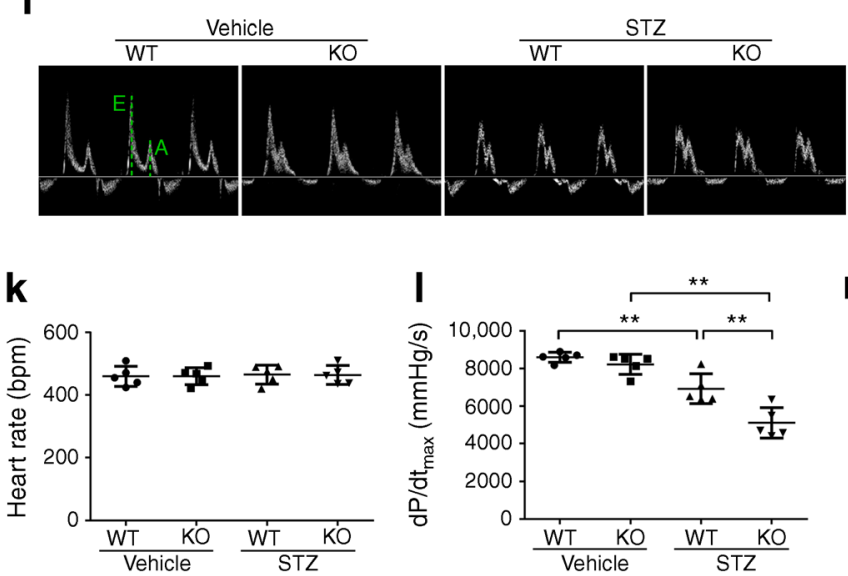

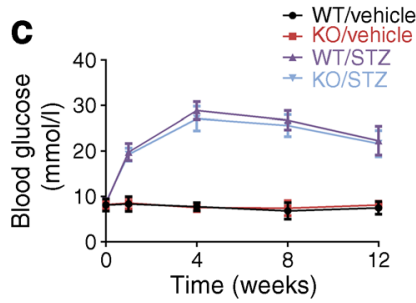

e
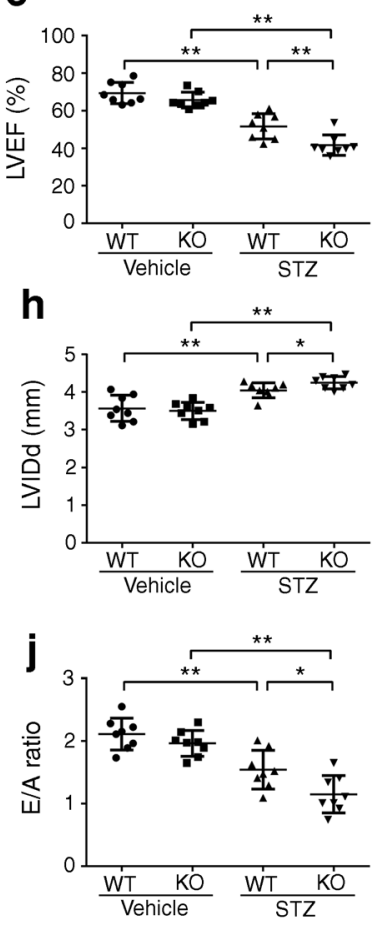

m

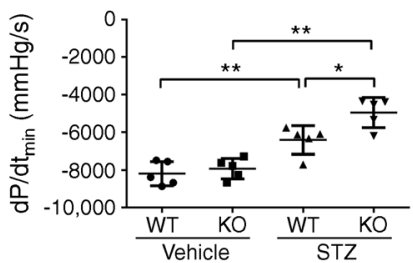

parameters of the mice are shown in ESM Table 3. Taken together, our data indicate that Akapl knockout exacerbates diabetes-induced cardiac dysfunction and myocardial fibrosis.

\section{Akap1 deficiency impairs mitochondrial respiratory function} Considering that cardiac dysfunction is closely correlated with reduced ATP synthesis and mitochondrial dysfunction $[6,20]$, we first examined mitochondrial morphology with TEM and detected mitochondrial membrane potential (one of the most important parameters for assessing mitochondrial function) by JC-1 staining. TEM analysis showed that mitochondria from hearts of mouse models of diabetes had reduced electron density. More specifically, mitochondria from the hearts of KO/STZ exhibited more obvious swelling (Fig. 3a). The mean mitochondrial size from the hearts of $\mathrm{KO} / \mathrm{STZ}$ was larger than that from WT/STZ. Furthermore, TEM analysis at higher magnification showed a decreased number of mitochondrial cristae, reduced cristae areas and an increased proportion of mitochondria with disorganised cristae in KO/STZ vs WT/ STZ (Fig. 3b-d). Moreover, the mitochondrial membrane potential was significantly decreased in primary neonatal cardiomyocytes transfected with siAkapl under high-glucose conditions, when compared with the control group (Fig. 3e, f). Given these profound changes in mitochondrial structure and mitochondrial membrane potential, we next analysed mitochondrial OCR to assess mitochondrial respiratory capacity. As expected, when compared with cardiomyocytes treated with control siRNA under high-glucose conditions and siAkapl-treated cardiomyocytes under normal-glucose conditions AKAP1 knockdown significantly suppressed 
Fig. 3 Akapl deficiency impairs mitochondrial respiratory function in the heart of diabetic mice. (a) Representative transmission electron microscopic images of mitochondria in WT and knockout $(\mathrm{KO})$ mice treated with vehicle or STZ (magnification $\times 6000[6 \mathrm{~K}]$ and $\times 16,000[16 \mathrm{~K}])$ and quantitative analysis of mitochondrial size. Scale bar at $6 \mathrm{~K}, 2 \mu \mathrm{m}$; scale bar at 16K, $1 \mu \mathrm{m}$. (b-d) Quantitative analysis of mitochondrial cristae number, cristae area and the proportion of mitochondria with disorganised cristae. In (a-d), $n=$ 8 mice. (e, f) Flow cytometry analysis and quantification of mitochondrial membrane potential by JC- 1 staining in primary neonatal cardiomyocytes. A high level of green fluorescence $(x$-axis) represents reduced mitochondrial membrane potential $(\Delta \Psi \mathrm{m})$ and a high level of red fluorescence ( $y$-axis) represents normal $\Delta \Psi \mathrm{m}$. The red fluorescence rate was analysed. $n=5$ wells. $(\mathbf{g}, \mathbf{h})$ Measure of OCR and respective quantitative analysis in primary neonatal cardiomyocytes; $n=5$ wells. FCCP, trifluoromethoxy carbonyl cyanide phenylhydrazone; $\mathrm{HG}$, high glucose (30 mmol/l); $\mathrm{NG}$, normal glucose $(5.5 \mathrm{mmol} / \mathrm{l})$; siCtrl, control small interfering RNA. Data are shown as mean \pm SD. $* p<0.05, * * p<0.01$ a
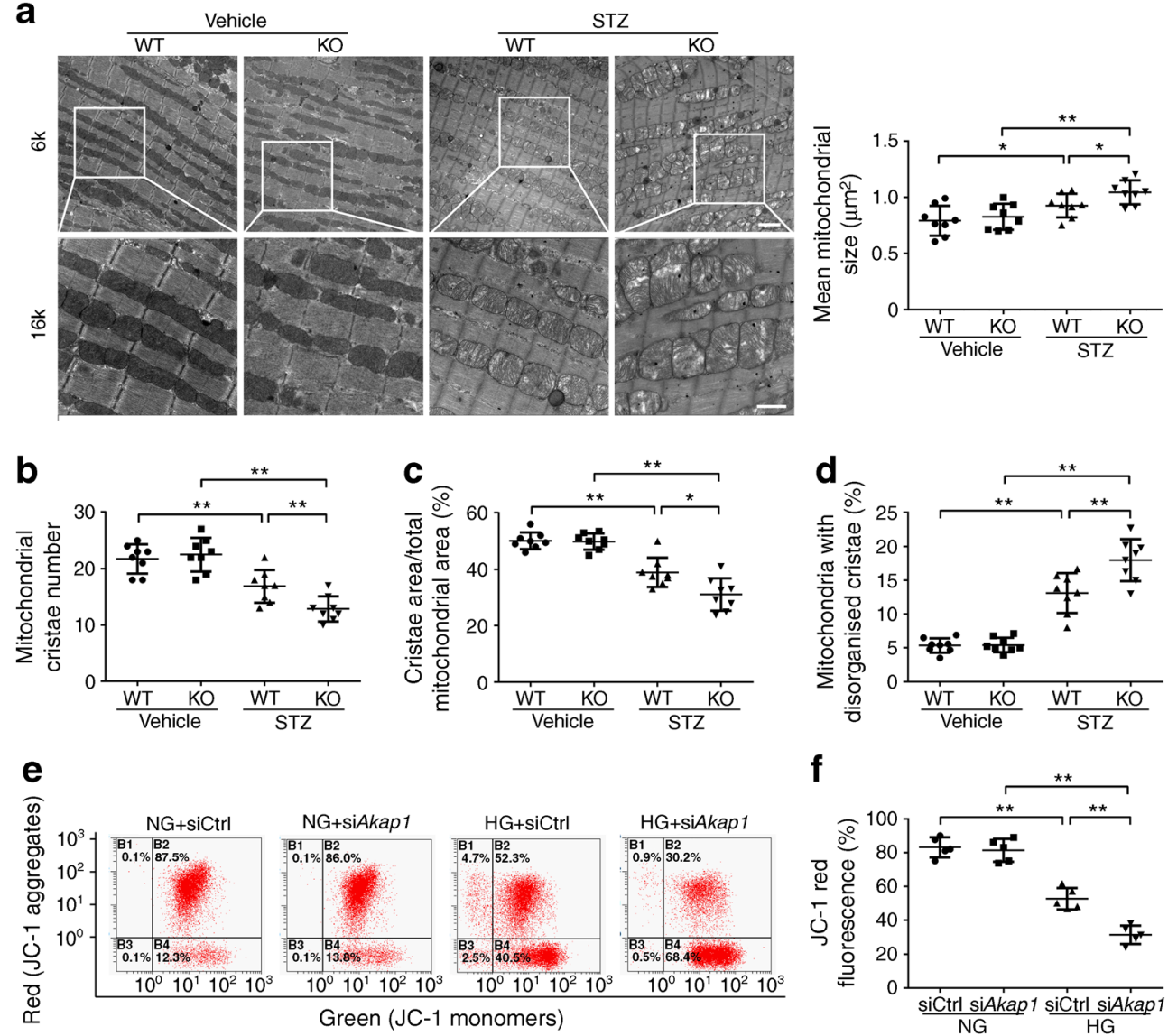

f

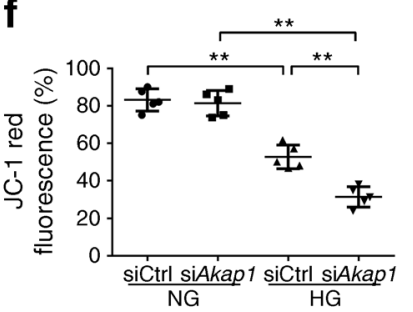

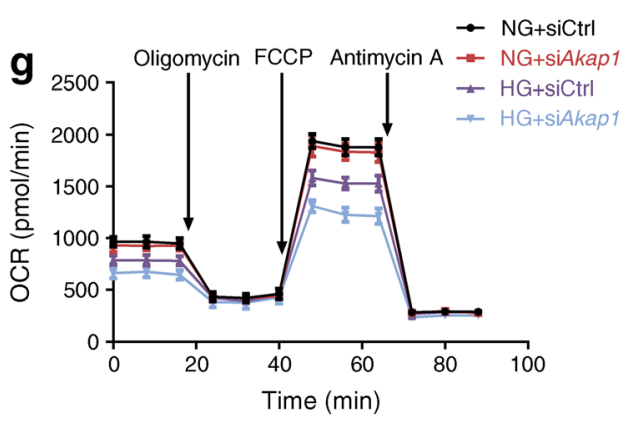

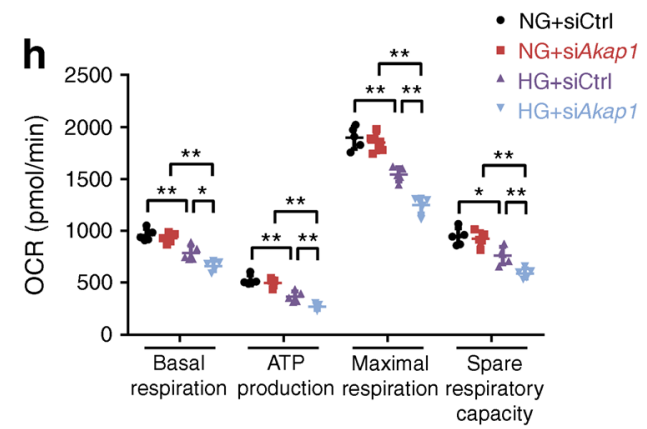

mitochondrial respiratory capacity, including basal respiration, ATP production, maximal respiration and spare respiration, in high-glucose-treated cardiomyocytes (Fig. 3g, h). Taken together, our data indicate that mitochondrial function is impaired in the hearts of diabetic mice and high-glucosetreated cardiomyocytes, and Akapl deficiency further exacerbates mitochondrial dysfunction.

\section{Akap1 deficiency increases mitochondrial ROS to aggravate cardiomyocyte apoptosis in diabetic hearts Mitochondrial dysfunction is often accompanied by changes in mitochondri- al ROS levels [5]. Therefore, we first detected mitochondrial ROS production in mouse hearts. Our data demonstrated that Akapl deficiency increased mitochondrial ROS production in}

diabetic hearts (Fig. 4a). Previous studies have reported that excessive ROS production easily leads to apoptosis [16, 22]. As expected, TUNEL staining analysis demonstrated that the number of apoptotic cells was significantly increased in hearts from KO/STZ when compared with those from WT/STZ (Fig. 4b). Moreover, Akapl deficiency further significantly increased the expression level of cleaved caspase- 3 in hearts from KO/STZ mice vs WT/STZ mice (Fig. 4c). Consistently, AKAP1 knockdown in primary neonatal cardiomyocytes significantly promoted cell apoptosis under high-glucose conditions when compared with the control group (Fig. 4d, e). Collectively, our data indicate that Akapl deficiency increases mitochondrial ROS to aggravate cardiomyocyte apoptosis in hearts of diabetic mice. 
Fig. 4 Akapl deficiency increases mitochondrial ROS to aggravate cardiomyocyte apoptosis in diabetic mice. (a) Myocardial mitochondrial ROS levels were analysed by confocal microscopy after staining of WT or knockout (KO) mouse samples with MitoSOX. Representative confocal microscope images and quantitative analysis of mitochondrial ROS production (MitoSOX fluorescence; red) are presented. Scale bar, $50 \mu \mathrm{m} ; n=5$ mice. (b) TUNEL assay by double staining with DAPI (blue) and TUNEL (green) detected apoptotic cells in mouse hearts. The quantification of TUNELpositive nuclei is shown. Scale bar, $50 \mu \mathrm{m} ; n=5$ mice. (c) Representative western blot images of cleaved caspase-3, together with quantification; $n=3$ mice. (d, e) Flow cytometry analysis of apoptosis by Annexin V-FITC and propidium iodide (PI) staining and quantification of apoptotic cells in primary neonatal cardiomyocytes with treatment as indicated; $n=6$ wells. Data are shown as mean \pm SD. HG, high glucose $(30 \mathrm{mmol} /$ 1); NG, normal glucose (5.5 mmol/l); siCtrl, control small interfering RNA. ${ }^{*} p<0.05$, $* * p<0.01$
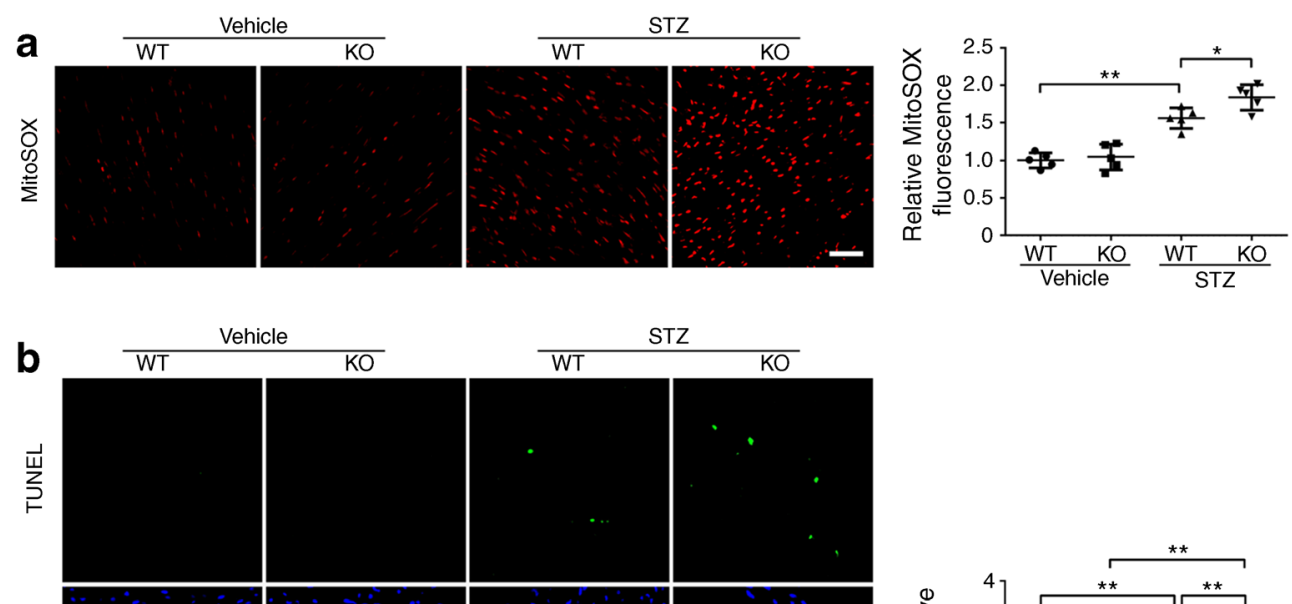

STZ

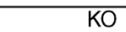

亨
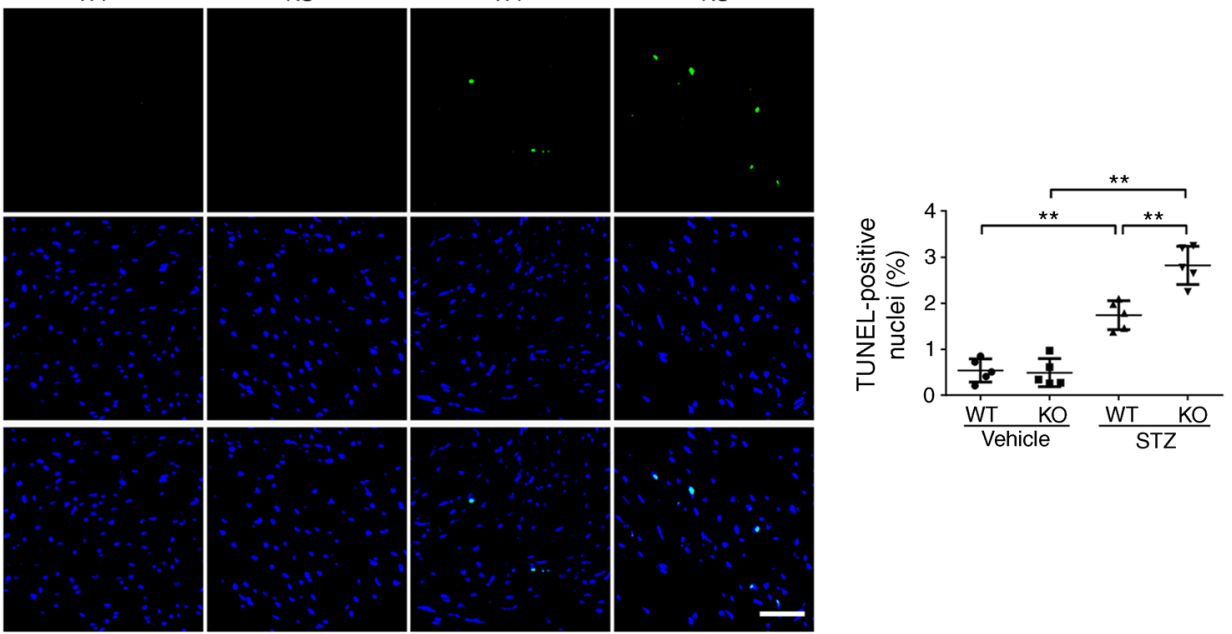

C
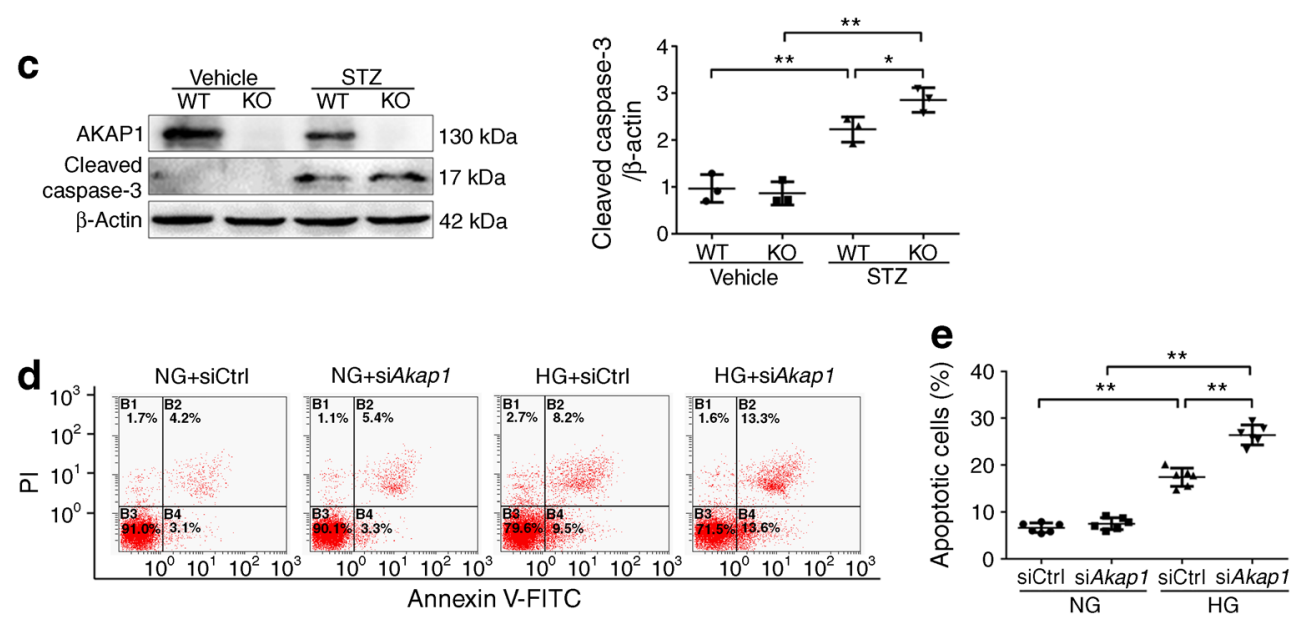

\begin{abstract}
AKAP1 promotes translocation of the NADH-ubiquinone oxidoreductase $75 \mathrm{kDa}$ subunit into mitochondria to regulate complex I activity To further explore the mechanism of AKAP1 in diabetic cardiomyopathy, we performed Co-IP assays. The experimental process is shown in Fig. 5a. The silver staining results showed a strong AKAP1 band around $130 \mathrm{kDa}$. There was also a band between $70 \mathrm{kDa}$ and $100 \mathrm{kDa}$, which might contain several AKAP1-interacting proteins (Fig. 5b). Following LC-MS/MS analysis of the target band, the AKAP1-interacting protein NADH-ubiquinone oxidoreductase $75 \mathrm{kDa}$ subunit (NDUFS1) was selected for further analysis (Table 1).
\end{abstract}

To further verify the interaction between AKAP1 and NDUFS1 in diabetic cardiomyopathy, we designed several additional experiments. First, we performed immunoprecipitation (IP) assays with different antibodies. As expected, an obvious interaction between AKAP1 and NDUFS1 was detected (Fig. 5c, d). Next, the interaction weakened or even disappeared in KO/STZ vs WT/STZ (Fig. 5e). Meanwhile, immunofluorescence was performed to show the colocalisation of AKAP1 and NDUFS1 in primary neonatal cardiomyocytes (Fig. 5f). Interestingly, as shown by western blotting (Fig. 5g), total NDUFS1 expression was not different in cardiomyocytes treated with siAkapl and cardiomyocytes 


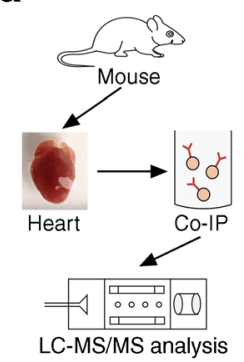

d

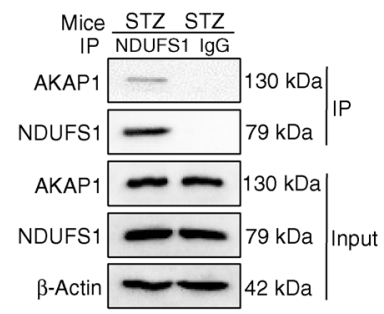

b

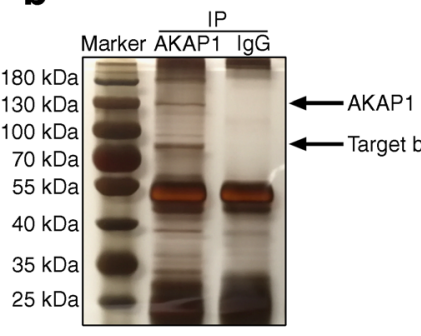

C

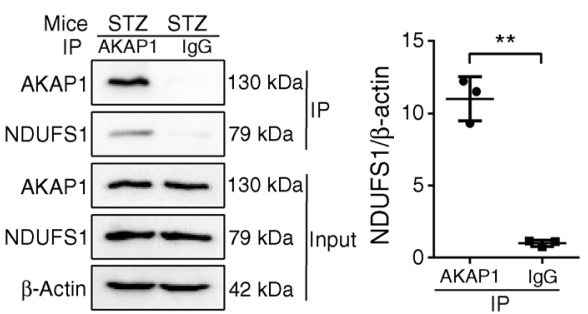

f
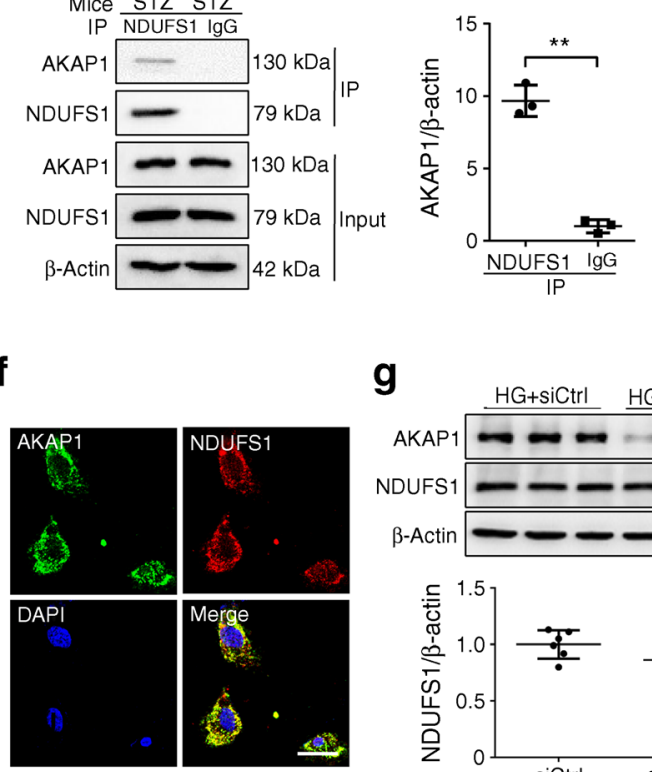

e

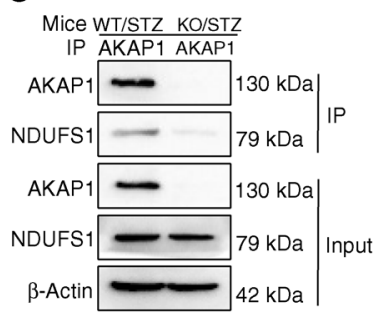

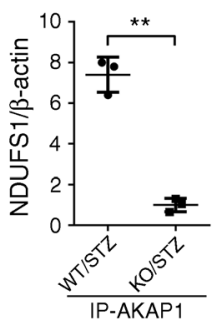

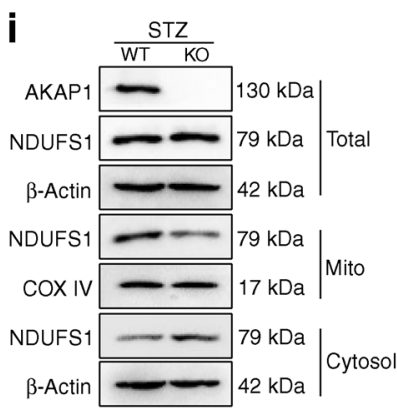

g
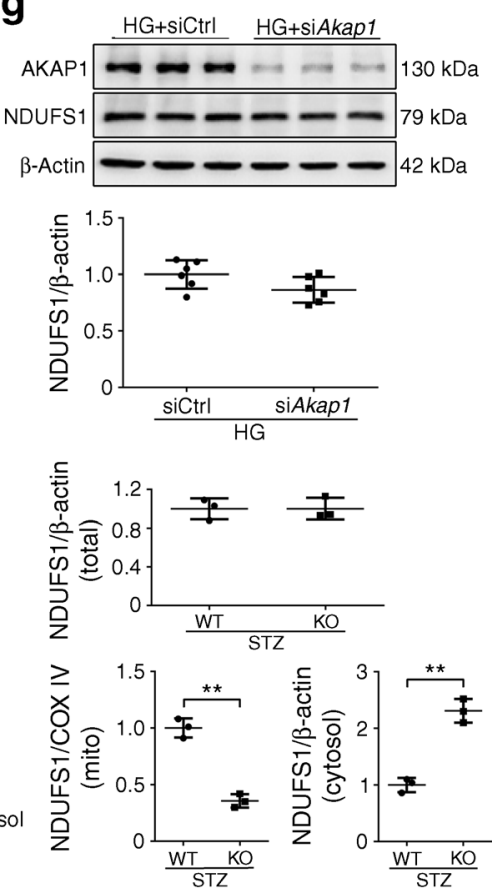

Fig. 5 AKAP1 promotes translocation of NDUFS1 into mitochondria to regulate complex I activity. (a) Schematic representation of the experimental protocol. (b) Immunoprecipitation (IP) assay was carried out using AKAP1 antibody or IgG (negative control antibody). Samples were electrophoresed and silver stained. Arrows indicate AKAP1 and the target bands; $n=3$ mice. (c, d) Interaction between AKAP1 and NDUFS1 was demonstrated via Co-IP; $n=3$ mice. (e) Co-IP of AKAP1 and NDUFS1 in STZ-treated WT and KO mice; $n=3$ mice. (f) Representative images showing immunofluorescence staining for AKAP1 (green), NDUFS1

treated with control siRNA. Considering that NDUFS1 is synthesised in the cytosol and then transferred into mitochondria, cytosol and mitochondria from hearts of diabetic mice were separated. We found that Akapl deficiency significantly

h
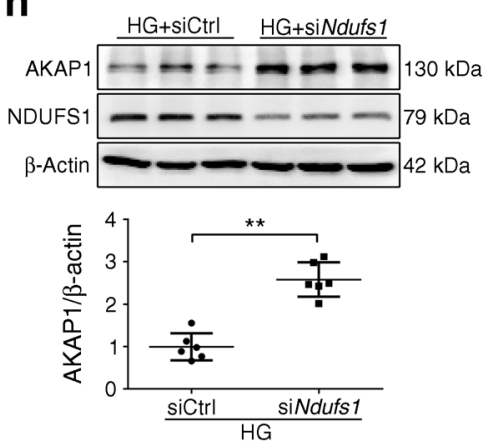

j

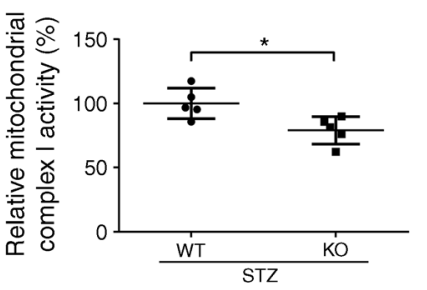

(red) and DAPI (blue) in primary neonatal cardiomyocytes. Scale bar, $25 \mu \mathrm{m} ; n=3$ wells. (g, h) Western blot demonstrating AKAP1 and NDUFS1 knockdown efficiency, with quantifications below; $n=6$ wells. (i) Western blot demonstrating the difference in cellular distribution of NDUFS1 between WT/STZ and KO/STZ, with quantifications to the right; $n=3$ mice. (j) Analysis of complex I activity in WT and knockout (KO) mice; $n=5$ mice. Data are shown as mean $\pm \mathrm{SD}$. COX IV, mitochondrial cytochrome c oxidase subunit IV; Mito, mitochondria; siCtrl, control small interfering RNA. * $p<0.05, * * p<0.01$

blocked the translocation of NDUFS1 into mitochondria without affecting total cellular NDUFS1 expression (Fig. 5i). Consistently, the immunofluorescence results showed that AKAP1 knockdown disrupted the co-localisation of 
Table 1 Mass spectrum analysis of AKAP1-interacting proteins

\begin{tabular}{llllr}
\hline Protein name & Unique peptides & Molecular mass $(\mathrm{kDa})$ & Abundance & MS score \\
\hline NDUFS1 & 33 & 79.7 & $2.5 \times 10^{8}$ & 182 \\
IMMT & 21 & 83.8 & $1.3 \times 10^{7}$ & 59 \\
PCCA & 19 & 79.9 & $1.3 \times 10^{7}$ & 49 \\
TRAP1 & 14 & 80.1 & $6.3 \times 10^{6}$ & 38 \\
PFKM & 12 & 85.2 & $3.9 \times 10^{6}$ & 32 \\
\hline
\end{tabular}

Following Co-IP assays of mouse heart tissue and visualisation by electrophoresis, the target band (AKAP1interacting proteins, between 70 and $100 \mathrm{kDa}$ ) was excised and assessed by LC-MS/MS. IMMT, inner membrane mitochondrial protein; PCCA, propionyl-CoA carboxylase alpha chain, mitochondrial; PFKM, ATP-dependent 6phosphofructokinase, muscle type; TRAP1, tumour necrosis factor receptor associated protein 1
Fig. 6 Cardiac overexpression of Akapl in the heart restores mitochondrial respiratory function via AKAP1-mediated NDUFS1 translocation. (a) Western blot showing cellular NDUFS1 distribution, with quantifications to the right; $n=3$ mice. (b) Analysis of complex I activity in WT and knockout (KO) mice, treated as indicated; $n=5$ mice. (c, d) OCR and associated quantitative analysis in primary neonatal cardiomyocytes, with treatment as indicated; $n=5$ wells. (e) Representative transmission electron microscopic images of mitochondria (magnification $\times 6000[6 \mathrm{~K}]$ and $\times 16000[16 \mathrm{~K}])$ and quantitative analysis of the mitochondrial size. Scale bar at $6 \mathrm{~K}, 2 \mu \mathrm{m}$; scale bar at $16 \mathrm{~K}, 1 \mu \mathrm{m}$. (f-h) Quantitative analysis of mitochondrial cristae number, cristae area and the proportion of mitochondria with disorganised cristae. In (e-h) $n=$ 8 mice. Data are shown as mean \pm SD. COX IV, mitochondrial cytochrome c oxidase subunit IV; FCCP, trifluoromethoxy carbonyl cyanide phenylhydrazone; HG, high glucose (30 mmol/l); Mito, mitochondria; siCtrl, control small interfering RNA. * $p<0.05$, $* * p<0.01$
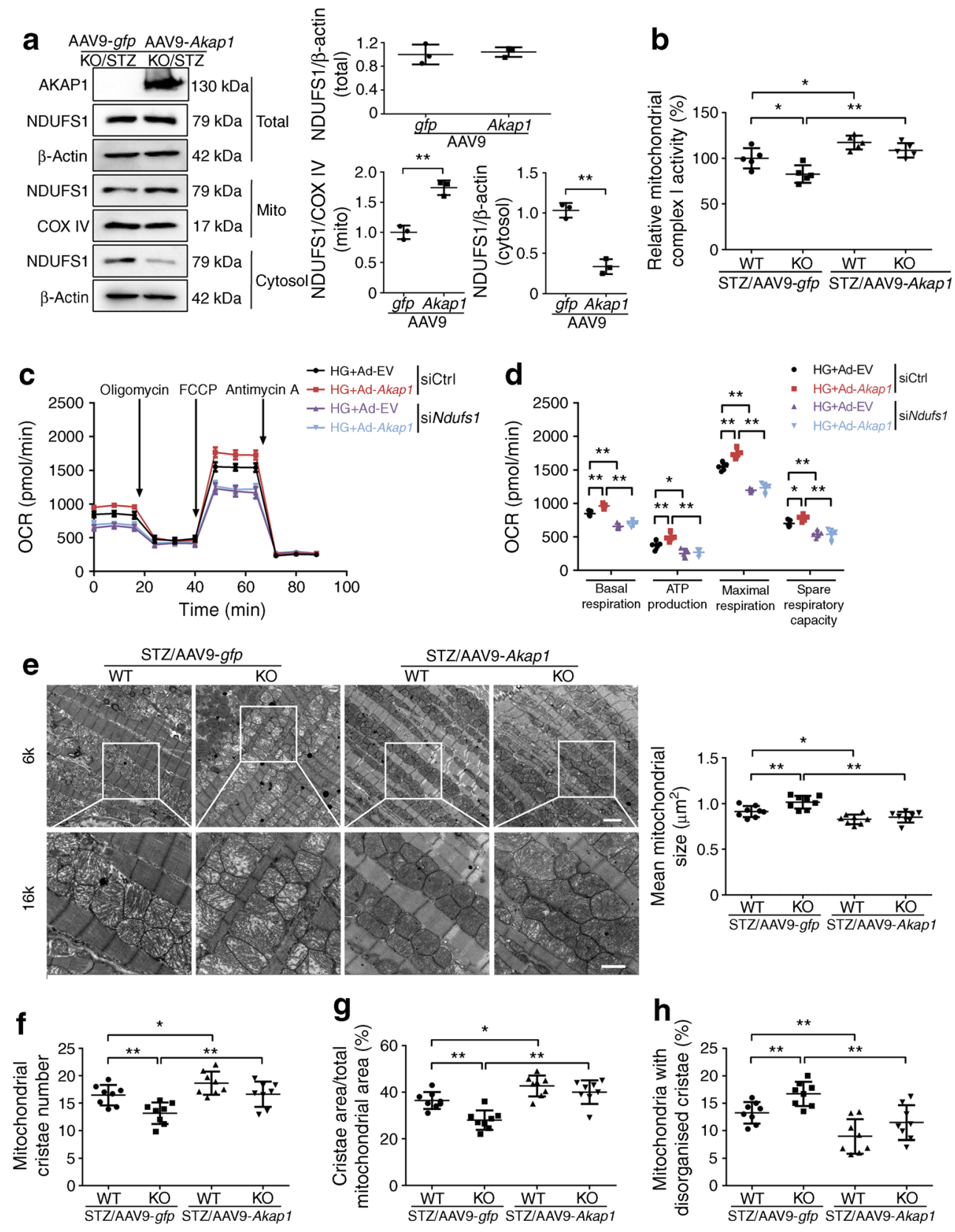
Fig. 7 Overexpression of Akap 1 decreases mitochondrial ROS to reduce cardiomyocyte apoptosis. (a) Representative confocal microscope images and quantitative analysis of mitochondrial ROS production (MitoSOX fluorescence; red) after staining of WT or knockout (KO) mouse samples with MitoSOX. Scale bar, $50 \mu \mathrm{m} ; n=$ 5 mice. (b) TUNEL assay by double staining with DAPI (blue) and TUNEL (green) detected apoptotic cells in mouse hearts. The quantification of TUNELpositive nuclei is shown. Scale bar, $50 \mu \mathrm{m} ; n=5$ mice. (c) Representative western blotting images of cleaved caspase-3, together with quantification; $n=3$ mice. (d, e) Flow cytometry analysis of apoptosis by Annexin V-FITC and propidium iodide (PI) staining and quantification of apoptotic cells in primary neonatal cardiomyocytes, with treatment as indicated; $n=6$ wells. Data are shown as mean \pm SD. siCtrl, control small interfering RNA. ${ }^{*} p<0.05$, $* * p<0.01$
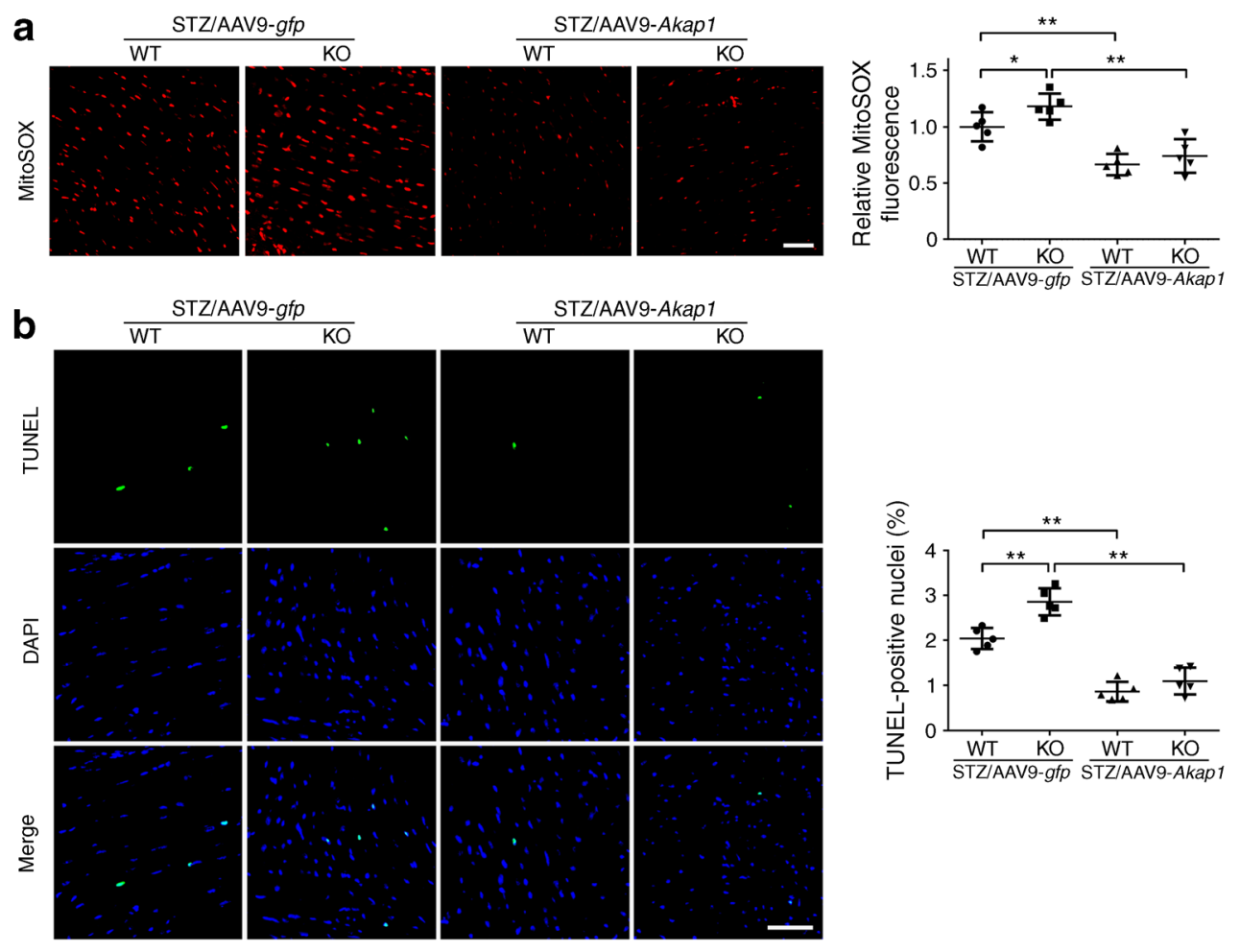

C
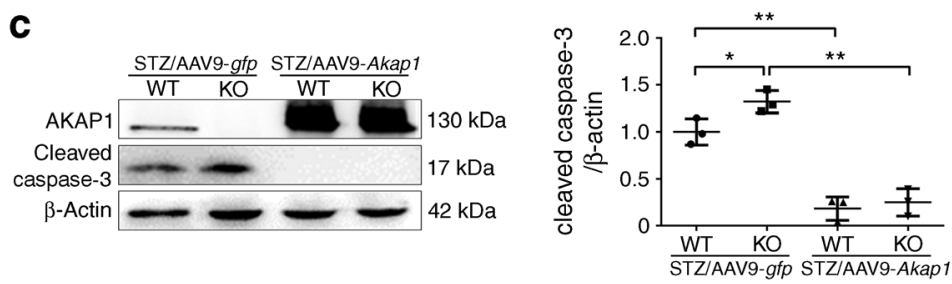

d
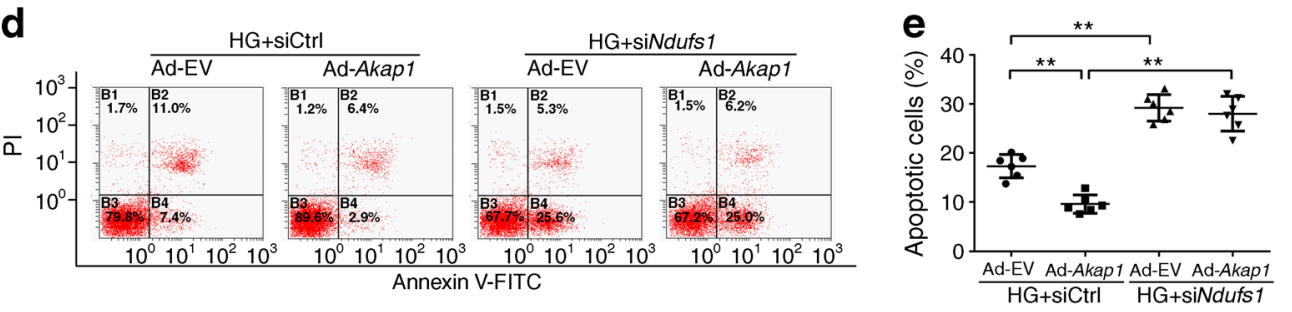

NDUFS1 and mitochondria (labelled with MitoTracker) (ESM Fig. 3a). As a core subunit of mitochondrial complex I, NDUFS1 plays a central role in regulating mitochondrial complex I activity. As expected, Akapl deficiency decreased complex I activity in KO/STZ when compared with WT/STZ mice (Fig. 5j). Collectively, our data demonstrate that Akap1 deficiency effects intracellular translocation of NDUFS1 in diabetic cardiomyopathy.

\section{Cardiac overexpression of Akap1 restores mitochondrial} respiratory function via AKAP1-mediated NDUFS1 translocation To further demonstrate the role of AKAP1 in diabetic cardiomyopathy, we restored AKAP1 expression by injection of AAV9-Akapl in mouse hearts. Cardiac-specific overexpression of Akapl was verified by western blotting (ESM Fig. 4a, b). We found that overexpression of Akapl promoted translocation of NDUFS1 from the cytosol to mitochondria (Fig. 6a). Similarly, complex I activity in hearts of diabetic mice following injection with AAV9-Akapl was increased when compared with those injected with adenoassociated virus carrying $g f p$ (AAV9-g $f p$; Fig. 6b). To further confirm that the effect of Akapl on cardiac function was mediated by NDUFS1, mitochondrial function was analysed by JC-1 staining and a mitochondrial OCR assay. AKAP1 was overexpressed in primary neonatal cardiomyocytes infected with Ad-Akapl when compared with the Ad-gfp group (ESM Fig. 4c, d). The mitochondrial membrane potential was rescued after Akapl overexpression, but the protective 
Fig. 8 Overexpression of Akapl in the heart alleviates diabetesinduced cardiac dysfunction. (a) Representative echocardiography images from WT and knockout (KO) mice. LVIDd and LVIDs are labelled. (b-e) Echocardiography data analysis; $n=8$ mice. (f, $\mathbf{g}$ ) Representative Doppler echocardiography images and quantification of the early mitral diastolic wave/late mitral diastolic wave ratio (E/A ratio); $n=8$ mice. (h-j) Haemodynamic analysis. Peak derivative of pressure over time $\left(\mathrm{dP} / \mathrm{dt}_{\max }\right)$ is the maximal value of the instantaneous first derivative of left ventricular pressure; minimum peak derivative of pressure over time $\left(\mathrm{dP} / \mathrm{dt}_{\min }\right)$ is the minimum value of the instantaneous first derivative of left ventricular pressure; $n=5$ mice. Data are shown as mean $\pm \mathrm{SD}$. $* p<0.05$, $* * p<0.01$ a

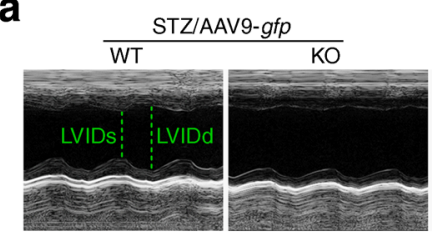

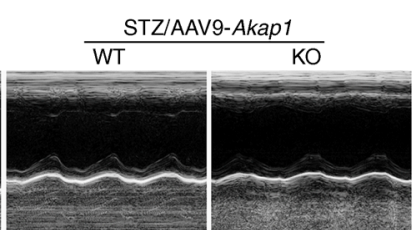
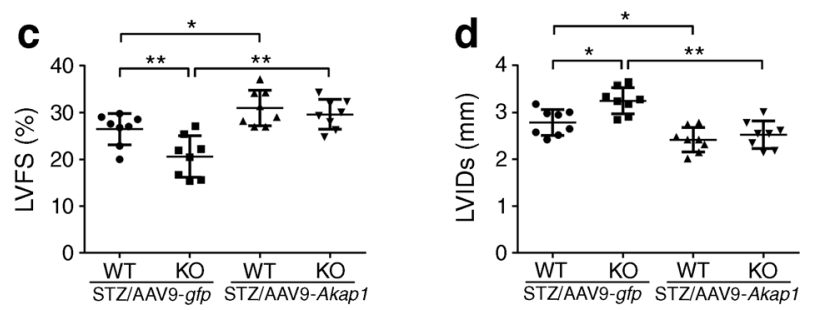

f
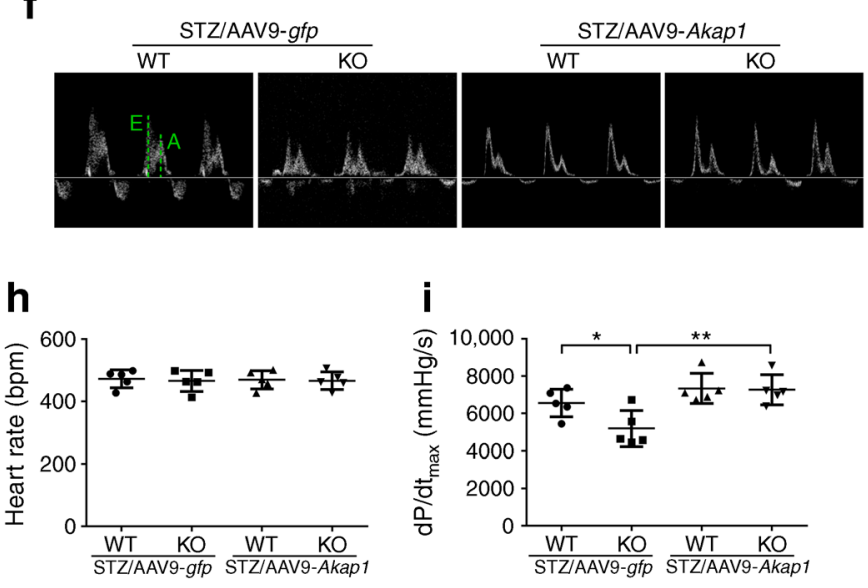
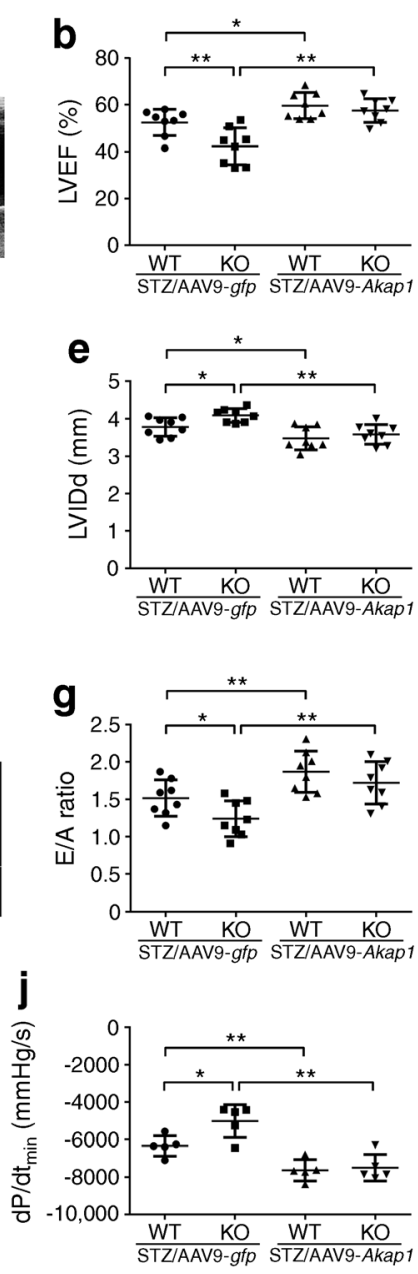

effect was blocked when Ndufs 1 was knocked down (ESM Fig. 4e, f). Consistent with the above-mentioned results, Akapl overexpression restored mitochondrial respiratory function, including basal respiration, ATP production and maximum respiration, but NDUFS1 knockdown blocked these phenomena (Fig. 6c, d). Additionally, TEM analysis demonstrated that cardiac-specific Akapl overexpression restored normal mitochondrial morphology in hearts of diabetic mice vs AAV9-gfp treated controls (Fig. 6e-h). Together, these data show that Akapl restoration rescues mitochondrial respiratory function via AKAP1-mediated NDUFS1 translocation.

\section{Overexpression of Akap1 decreases mitochondrial ROS to} reduce cardiomyocyte apoptosis Akapl overexpression reduced mitochondrial ROS production in the hearts of diabetic mice when compared with the control group (Fig. 7a). Apoptosis was reduced in WT/STZ injected with AAV9Akapl vs WT/STZ injected with AAV9-g $f p$. In addition, cell apoptosis was also reduced in KO/STZ injected with AAV9Akapl vs KO/STZ injected with AAV9-gfp (Fig. 7b). Moreover, Akapl overexpression significantly decreased the expression level of cleaved caspase-3 in hearts of mice with diabetes vs controls (Fig. 7c). The body weight and heart weight of mice that received AAV9-Akapl injection are shown in ESM Table 4. Consistent with the in vivo experiments, the cell apoptotic rate in cardiomyocytes was reduced after infection with Ad-Akapl (Fig. 7d, e). Akapl overexpression in primary neonatal cardiomyocytes significantly decreased mitochondrial ROS production under highglucose conditions, however, NDUFS1 knockdown blocked the protective role of AKAP1 in these cells (ESM Fig. 5a).

Overexpression of Akap1 in the heart alleviates diabetesinduced cardiac dysfunction To determine whether Akapl overexpression can rescue cardiac function, we overexpressed Akapl in mouse hearts by injection with AAV9-Akap1. As expected, Akapl overexpression improved myocardial contractile function and restored cardiac structure, as evidenced by increased LVEF (WT/STZ injected with AAV9-gfp vs WT/STZ injected with AAV9-Akap1, 52.4\% vs $59.6 \%$; KO/STZ injected with AAV9- $g f p$ vs $\mathrm{KO} / \mathrm{STZ}$ injected with AAV9-Akap1, 42.2\% vs $57.6 \%$ ), and other parameters, including increased LVFS and decreased LVIDs 
Fig. 9 Schematic diagram depicting mechanisms by which Akapl deficiency exacerbates diabetic cardiomyopathy by inducing NDUFS1-mediated mitochondrial dysfunction and apoptosis. Under the healthy condition, AKAP1 promotes translocation of NDUFS1 into mitochondria to regulate complex I activity. Under diabetic conditions, myocardial AKAP1 expression is decreased. Downregulation of Akapl blocks the translocation of NDUFS1 from the cytosol to mitochondria. Reduction of NDUFS1 in mitochondria decreases ATP production and increases mitochondrial ROS level, which causes mitochondrial dysfunction and cell apoptosis, respectively, thereby leading to cardiac dysfunction

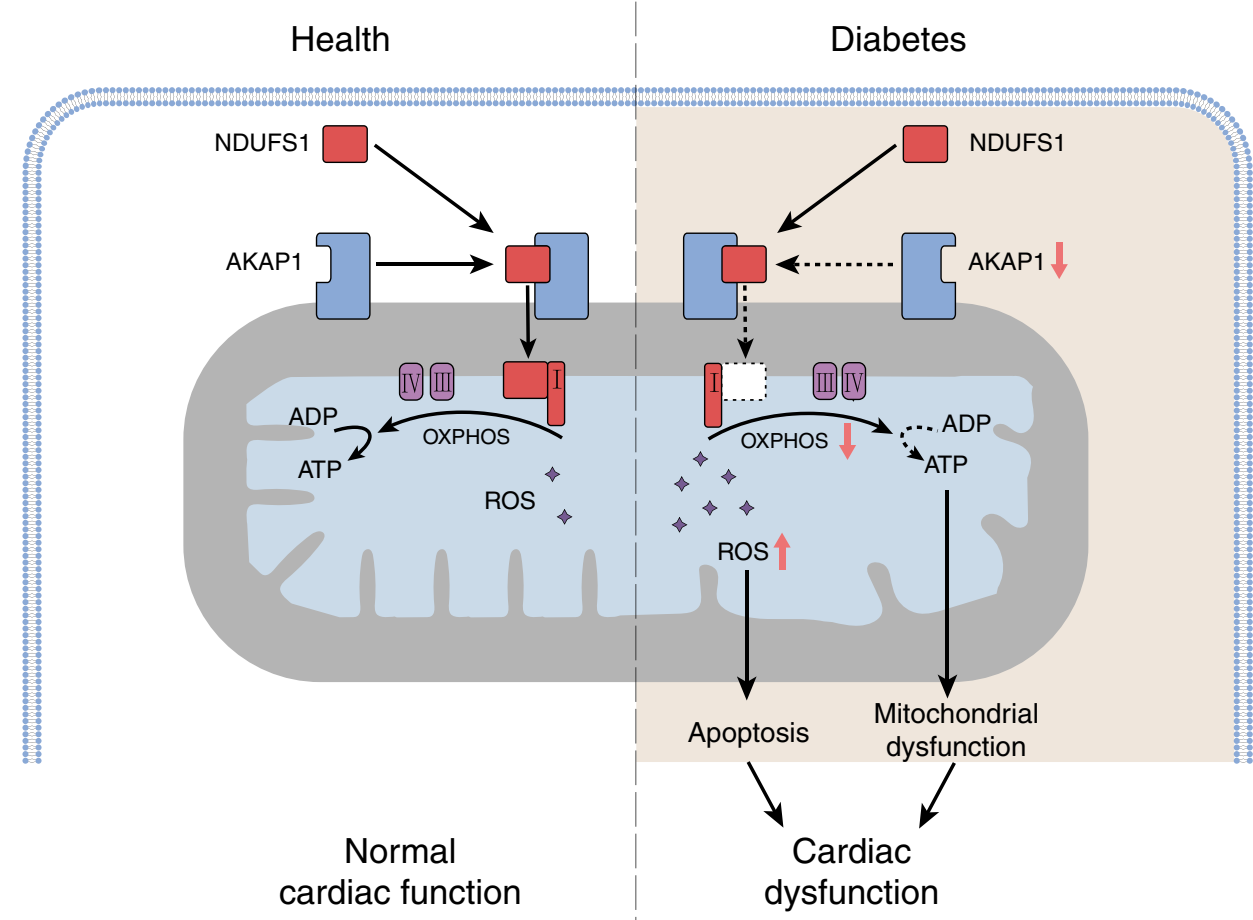

and LVIDd (Fig. 8a-e). Meanwhile, myocardial diastolic function was restored, as evidenced by the increased $\mathrm{E} / \mathrm{A}$ ratio in AAV9-Akap1-treated WT or Akap1-KO mice and their respective AAV9-gfp treated controls (Fig. 8f, g). The haemodynamic analysis also confirmed the restoration of cardiac function in hearts with AAV9-Akapl injection (Fig. 8h-j). Other parameters of echocardiography and haemodynamics are shown in ESM Fig. 6. Additionally, Akapl restoration in the hearts of WT and Akapl-KO mice with diabetes reduced the interstitial fibrosis content vs respective AAV9-gfp treated controls (ESM Fig. 7a). Taken together, our data show that cardiac-specific overexpression of Akapl alleviates diabetesinduced cardiac dysfunction and myocardial fibrosis.

\section{Discussion}

There were several important findings in our study. First, we found that AKAP1 was downregulated in the hearts of STZinduced diabetic mice. Second, Akapl deficiency in diabetic mice exacerbated cardiac dysfunction, which was accompanied by impaired mitochondrial function and increased cardiomyocyte apoptosis. Third, AKAP1 bound with NDUFS1 and promoted translocation of NDUFS1 to mitochondria to regulate complex I activity. In contrast, AKAP1 deficiency blocked NDUFS1 translocation from the cytosol to mitochondria, which inhibited OXPHOS and increased mitochondrial ROS production. Furthermore, cardiac-specific Akapl overexpression alleviated diabetic cardiomyopathy through improved mitochondrial function and reduced cardiomyocyte apoptosis. Collectively, our study demonstrated that Akapl deficiency exacerbated diabetic cardiomyopathy by NDUFS1-mediated mitochondrial dysfunction and apoptosis (Fig. 9). In addition, overexpression of Akapl in the hearts of diabetic mice alleviated diabetic cardiomyopathy. Therefore, targeting AKAP1 provides the possibility of a new therapeutic approach for diabetic cardiomyopathy.

Mitochondria are the main producers of ATP and the foremost source of intracellular ROS. As a mitochondrial outermembrane anchoring protein, AKAP1 plays an important role in regulating mitochondrial function and mitochondrial ROS production. A previous study has reported that Akapl-KO mice exposed to hyperoxia displayed disorganised abnormal mitochondria in an acute lung injury model [23]. Similarly, our data demonstrate that Akapl deficiency in diabetic mice impairs mitochondrial cristae and aggravates mitochondrial dysfunction. Another recent study has demonstrated that AKAP1 protects neuronal cells from glutamate-induced oxidative stress [24]. The study supports our results that Akapl deficiency in diabetic mice further increases mitochondrial ROS production, thereby aggravating cardiomyocyte apoptosis.

Mitochondrial oxidative damage and mitochondrial dysfunction contribute to the progression of diabetic cardiomyopathy [25-27]; however, the cause of mitochondrial dysfunction and the source of mitochondrial ROS were largely unclear [28]. To elucidate the molecular mechanism by which AKAP1 affects diabetic cardiomyopathy, we performed LC-MS/MS analysis. Co-IP and immunofluorescence assays demonstrated that NDUFS1 interacted with AKAP1 in cardiomyocytes. As a core subunit of 
mitochondrial complex I, NDUFS1 controls mitochondrial OXPHOS and mitochondrial ROS production [29, 30]. Our study found that Akapl deficiency caused NDUFS1 retention in the cytosol and blocked its translocation into mitochondria, which resulted in increased mitochondrial ROS production and impaired mitochondrial function. Several studies have also found that translocation of NDUFS1 plays a pivotal role in mitochondrial ROS production and cell apoptosis [29, 31]. NDUFS1 knockdown in primary neonatal cardiomyocytes enhanced the expression of AKAP1 that in turn promoted translocation of NDUFS1 into mitochondria (Fig. 5h, i, Fig. $6 a)$, which may be a negative feedback regulation to alleviate the decreased complex I. As a core subunit of complex I, NDUFS1 regulates mitochondrial function by regulation of complex I activity. Our data indicated that the translocation of NDUFS1 from the cytosol to mitochondria affected mitochondrial ROS production and oxygen consumption in diabetic cardiomyopathy, thereby affecting myocardial function.

Our previous studies have demonstrated that, in diabetes, the heart shows severe mitochondrial dysfunction and enhanced oxidative stress levels $[5,16]$. In this study, we demonstrate that the absence of AKAP1 is the primary cause of aggravated myocardial damage in diabetic cardiomyopathy progression. These results are similar to a previous study that reported that downregulation of Akapl is associated with mitochondrial dysfunction, high oxidative stress and apoptosis in a rat model of cardiac hypertrophy [14]. Nevertheless, another study reported that Akapl deletion increases cardiomyocyte mitophagy and apoptosis under conditions of myocardial ischaemia [32]. This difference suggests that AKAP1 may regulate cardiac function via multiple mechanisms, depending on different disease models.

Another important finding of this study is that Akapl overexpression in the hearts of mouse models of diabetes alleviates diabetic cardiomyopathy. Overexpression of Akapl by injection of AAV9-Akap1 increases NDUFS1 levels in mitochondria, which restores complex I activity, improves oxidative respiratory chain efficiency and reduces mitochondrial ROS production. Until now, multiple preclinical and clinical trials have shown that adeno-associated virus (AAV) is safe and effective in different diseases [33-35]. AAV9 is widely used for cardiac-specific gene delivery in mice and rats [36]. Given that impaired mitochondrial function and elevated mitochondrial ROS production are the main reasons for cardiac dysfunction in diverse heart diseases [37-39], targeting Akap1 using AAV9 might be a potential therapeutic strategy for the treatment of diabetic cardiomyopathy and related heart diseases.

Our study found that AKAP1 is decreased in the hearts of diabetic mice, which is similar to a previous study. The previous study reported that AKAP1 expression was downregulated in mice after myocardial infarction and the genetic deletion of Siah 2 prevented AKAP1 degradation after coronary artery ligation [32]. Another study has also demonstrated that AKAP1 expression level is regulated by siah E3 ubiquitin protein ligase 2 (SIAH2) under hypoxic conditions [10]. These findings suggest that the reduction of AKAP1 expression may be mediated by SIAH2 in diabetic cardiomyopathy, which requires further investigation. Several studies reported that cardiac function and mitochondrial respiration were not statistically different between WT mice and Akapl-KO mice in vehicle-treated groups $[32,40]$, which is similar with our study. In addition, no significant difference was observed in mitochondrial ROS production and apoptosis rate between vehicle-treated Akap1-KO mice and vehicle-treated WT mice. These findings indicate that mitochondrial capacity may be sufficient for maintaining myocardial energy and cardiac function when confronted with deletion of Akapl in healthy conditions. In contrast, mitochondria could not afford an increased workload in diabetes, and Akap 1 deficiency further aggravates mitochondrial dysfunction under strong stress, such as that induced by hyperglycaemia.

There are several limitations in this study. First, cardiomyocyte-specific Akap1-KO mice were not used in our study. To logically prove the experimental results, cardiacspecific Akap1 overexpression was conducted by injecting AAV9-Akap1 into hearts. In addition, our data were verified in primary neonatal cardiomyocytes through transfection with siAkap1 or via infection with Ad-Akap1. Second, due to ethical reasons, the expression level of cardiac AKAP1 could not be determined in individuals with diabetes. Third, in spite of STZinduced mice being used as the experimental diabetic model in multiple studies $[6,18,20]$, this model cannot completely mimic clinical diabetes in humans. Last, the specific dynamic process in diabetic cardiomyopathy by which AKAP1 promotes translocation of NDUFS1 into mitochondria is still unclear. Further study is needed to explain the interaction between AKAP1/NDUFS1 and to obtain clinical metabolic data in humans with diabetes. Despite these limitations, we believe that our study provides new insights into the role and mechanisms of action of AKAP1 in diabetic cardiomyopathy.

In summary, the novel findings in this study allow us to suggest a new mechanism by which AKAP1 promotes the translocation of NDUFS1 from the cytosol to mitochondria and, thus, alleviates diabetic cardiomyopathy. Considering that the expression of AKAP1 is downregulated in diverse heart diseases, AKAP1 restoration using an AAV9 approach provides compelling evidence for the possibility of a new therapeutic strategy for diabetic cardiomyopathy and related heart diseases.

Data availability The data are available on request from the authors.

Funding This work was supported by grants from the National Natural Science Foundation of China (No. 81772935, No. 81972590, No. 81400197, No. 81770369 and No. 81570252). 
Duality of interest The authors declare that there is no duality of interest associated with this manuscript.

Contribution statement $\mathrm{BQ}, \mathrm{LH}$ and $\mathrm{YZ}$ designed the experiments, acquired and analysed data and drafted the manuscript. LZ, YH, JL and $\mathrm{CL}$ contributed to the acquisition and analysis of data and drafted the manuscript. BZ, QH and JX contributed to the experimental design and interpretation of data, and drafted the manuscript. FL, YL and LJ designed the study, analysed the data and revised the manuscript. All of the authors have approved the final version to be published. LJ is responsible for the integrity of the work as a whole.

\section{References}

1. Zhou B, Lu Y, Hajifathalian K et al (2016) Worldwide trends in diabetes since 1980: A pooled analysis of 751 population-based studies with $4 \cdot 4$ million participants. Lancet 387(10027):15131530. https://doi.org/10.1016/S0140-6736(16)00618-8

2. Cho NH, Shaw JE, Karuranga S et al (2018) IDF Diabetes Atlas: global estimates of diabetes prevalence for 2017 and projections for 2045. Diabetes Res Clin Pract 138:271-281. https://doi.org/10. 1016/j.diabres.2018.02.023

3. Morrish NJ, Wang SL, Stevens LK, Fuller JH, Keen H (2001) Mortality and causes of death in the WHO Multinational Study of Vascular Disease in Diabetes. Diabetologia 44(Suppl 2):S14-S21. https://doi.org/10.1007/p100002934

4. Dillmann WH (2019) Diabetic cardiomyopathy. Circ Res 124(8): 1160-1162. https://doi.org/10.1161/CIRCRESAHA.118.314665

5. Hu L, Ding M, Tang D et al (2019) Targeting mitochondrial dynamics by regulating Mfn2 for therapeutic intervention in diabetic cardiomyopathy. Theranostics 9(13):3687-3706. https://doi.org/ $10.7150 /$ thno. 33684

6. Ding M, Feng N, Tang D et al (2018) Melatonin prevents Drp1mediated mitochondrial fission in diabetic hearts through SIRT1PGC1 $\alpha$ pathway. J Pineal Res 65(2):e12491. https://doi.org/10. 1111/jpi.12491

7. He Y, Zhou L, Fan Z, Liu S, Fang W (2018) Palmitic acid, but not high-glucose, induced myocardial apoptosis is alleviated by Nacetylcysteine due to attenuated mitochondrial-derived ROS accumulation-induced endoplasmic reticulum stress. Cell Death Dis 9(5):568. https://doi.org/10.1038/s41419-018-0593-y

8. Huynh K, Kiriazis H, Du XJ et al (2012) Coenzyme $\mathrm{Q}_{10}$ attenuates diastolic dysfunction, cardiomyocyte hypertrophy and cardiac fibrosis in the $\mathrm{db} / \mathrm{db}$ mouse model of type 2 diabetes. Diabetologia 55(5):1544-1553. https://doi.org/10.1007/s00125012-2495-3

9. Ansley DM, Wang B (2013) Oxidative stress and myocardial injury in the diabetic heart. J Pathol 229(2):232-241. https://doi.org/10. 1002/path.4113

10. Kim H, Scimia MC, Wilkinson D et al (2011) Fine-tuning of Drp1/Fis1 availability by AKAP121/Siah2 regulates mitochondrial adaptation to hypoxia. Mol Cell 44:532-544. https://doi.org/10. 1016/j.molcel.2011.08.045

11. Scorziello A, Savoia C, Sisalli MJ et al (2013) NCX3 regulates mitochondrial $\mathrm{Ca}^{2+}$ handling through the AKAP121-anchored signaling complex and prevents hypoxia-induced neuronal death. J Cell Sci 126:5566-5577. https://doi.org/10.1242/jcs.129668

12. Livigni A, Scorziello A, Agnese S et al (2006) Mitochondrial AKAP121 links cAMP and src signaling to oxidative metabolism. Mol Biol Cell 17(1):263-271. https://doi.org/10.1091/mbc.e05-090827
13. Carlucci A, Gedressi C, Lignitto L et al (2008) Protein-tyrosine phosphatase PTPD1 regulates focal adhesion kinase autophosphorylation and cell migration. J Biol Chem 283(16):10919-10929. https://doi.org/10.1074/jbc.M707248200

14. Perrino C, Feliciello A, Schiattarella GG et al (2010) AKAP121 downregulation impairs protective cAMP signals, promotes mitochondrial dysfunction, and increases oxidative stress. Cardiovasc Res 88(1):101-110. https://doi.org/10.1093/cvr/cvq155

15. Yan W, Guo Y, Tao L et al (2017) C1q/tumor necrosis FactorRelated protein-9 regulates the fate of implanted mesenchymal stem cells and mobilizes their protective effects against ischemic heart injury via multiple novel signaling pathways. Circulation 136(22): 2162-2177. https://doi.org/10.1161/CIRCULATIONAHA.117. 029557

16. Ji L, Liu F, Jing Z et al (2017) MICU1 alleviates diabetic cardiomyopathy through mitochondrial $\mathrm{Ca}^{2+}$-Dependent antioxidant response. Diabetes 66:1586-1600. https://doi.org/10.2337/db161237

17. Xuan F, Huang M, Zhao E, Cui H (2018) MINA53 deficiency leads to glioblastoma cell apoptosis via inducing DNA replication stress and diminishing DNA damage response. Cell Death Dis 9(11): 1062. https://doi.org/10.1038/s41419-018-1084-x

18. Wu S, Lu Q, Ding Y et al (2019) Hyperglycemia-Driven inhibition of AMP-Activated protein kinase $\alpha 2$ induces diabetic cardiomyopathy by promoting Mitochondria-Associated endoplasmic reticulum membranes in vivo. Circulation 139(16):1913-1936. https:// doi.org/10.1161/CIRCULATIONAHA.118.033552

19. Purvis G, Chiazza F, Chen J et al (2018) Annexin A1 attenuates microvascular complications through restoration of Akt signalling in a murine model of type 1 diabetes. Diabetologia 61(2):482-495. https://doi.org/10.1007/s00125-017-4469-y

20. Zhang M, Zhang L, Hu J et al (2016) MST1 coordinately regulates autophagy and apoptosis in diabetic cardiomyopathy in mice. Diabetologia 59(11):2435-2447. https://doi.org/10.1007/s00125016-4070-9

21. Teng X, Ji C, Zhong $\mathrm{H}$ et al (2019) Selective deletion of endothelial cell calpain in mice reduces diabetic cardiomyopathy by improving angiogenesis. Diabetologia 62(5):860-872. https://doi.org/10.1007/ s00125-019-4828-y

22. Bhatt NM, Aon MA, Tocchetti CG et al (2015) Restoring redox balance enhances contractility in heart trabeculae from type 2 diabetic rats exposed to high glucose. Am J Physiol Heart Circ Physiol 308:H291-H302. https://doi.org/10.1152/ajpheart.00378. 2014

23. Narala VR, Fukumoto J, Hernandez-Cuervo H et al (2018) Akap1 genetic deletion increases the severity of hyperoxia-induced acute lung injury in mice. Am J Physiol Lung Cell Mol Physiol 314(5): L860-L870. https://doi.org/10.1152/ajplung.00365.2017

24. Zhang J, Feng J, Ma D et al (2019) Neuroprotective mitochondrial remodeling by AKAP121/PKA protects HT22 cell from Glutamate-Induced oxidative stress. Mol Neurobiol 56(8):55865607. https://doi.org/10.1007/s12035-018-1464-3

25. Fillmore N, Mori J, Lopaschuk GD (2014) Mitochondrial fatty acid oxidation alterations in heart failure, ischaemic heart disease and diabetic cardiomyopathy. Br J Pharmacol 171(8):2080-2090. https://doi.org/10.1111/bph.12475

26. Kakoki M, Bahnson EM, Hagaman JR et al (2019) Engulfment and cell motility protein 1 potentiates diabetic cardiomyopathy via Racdependent and Rac-independent ROS production. JCI Insight 4(12):e127660. https://doi.org/10.1172/jci.insight.127660

27. Blake R, Trounce IA (2014) Mitochondrial dysfunction and complications associated with diabetes. Biochim Biophys Acta 1840(4):1404-1412. https://doi.org/10.1016/j.bbagen.2013.11.007

28. Faria A, Persaud SJ (2017) Cardiac oxidative stress in diabetes: mechanisms and therapeutic potential. Pharmacol Ther 172:50 62. https://doi.org/10.1016/j.pharmthera.2016.11.013 
29. Lopez-Fabuel I, Le Douce J, Logan A et al (2016) Complex I assembly into supercomplexes determines differential mitochondrial ROS production in neurons and astrocytes. Proc Natl Acad Sci U S A 113(46):13063-13068. https://doi.org/10.1073/pnas. 1613701113

30. Kussmaul L, Hirst J (2006) The mechanism of superoxide production by NADH: ubiquinone oxidoreductase (complex I) from bovine heart mitochondria. Proc Natl Acad Sci 103(20):76077612. https://doi.org/10.1073/pnas.0510977103

31. Elkholi R, Abraham-Enachescu I, Trotta AP et al (2019) MDM2 integrates cellular respiration and apoptotic signaling through NDUFS1 and the mitochondrial network. Mol Cell 74(3):452465. https://doi.org/10.1016/j.molcel.2019.02.012

32. Schiattarella GG, Cattaneo F, Pironti G et al (2016) Akap1 deficiency promotes mitochondrial aberrations and exacerbates cardiac injury following permanent coronary ligation via enhanced mitophagy and apoptosis. PLoS One 11:e154076. https://doi.org/ 10.1371/journal.pone.0154076

33. Hulot JS, Salem JE, Redheuil A et al (2017) Effect of intracoronary administration of AAV1/SERCA2a on ventricular remodelling in patients with advanced systolic heart failure: Results from the AGENT-HF randomized phase 2 trial. Eur J Heart Fail 19(11): 1534-1541. https://doi.org/10.1002/ejhf.826

34. Kojima K, Nakajima T, Taga N et al (2019) Gene therapy improves motor and mental function of aromatic 1-amino acid decarboxylase deficiency. Brain 142(2):322-333. https://doi.org/10.1093/brain/ awy331

35. Bennett J, Wellman J, Marshall KA et al (2016) Safety and durability of effect of contralateral-eye administration of AAV2 gene therapy in patients with childhood-onset blindness caused by RPE65 mutations: a follow-on phase 1 trial. Lancet 388:661-672. https://doi.org/10.1016/S0140-6736(16)30371-3

36. Bish LT, Morine K, Sleeper MM et al (2008) Adeno-associated virus (AAV) serotype 9 provides global cardiac gene transfer superior to AAV1, AAV6, AAV7, and AAV8 in the mouse and rat. Hum Gene Ther 19(12):1359-1368. https://doi.org/10.1089/hum.2008. 123

37. Sulaiman D, Li J, Devarajan A et al (2019) Paraoxonase 2 protects against acute myocardial ischemia-reperfusion injury by modulating mitochondrial function and oxidative stress via the PI3K/Akt/ GSK-3 $\beta$ RISK pathway. J Mol Cell Cardiol 129:154-164. https:// doi.org/10.1016/j.yjmcc.2019.02.008

38. Cao Y, Xu C, Ye J et al (2019) Miro2 regulates inter-mitochondrial communication in the heart and protects against TAC-induced cardiac dysfunction. Circ Res 125(125):728-743. https://doi.org/ 10.1161/CIRCRESAHA.119.315432

39. Dey S, DeMazumder D, Sidor A, Foster DB, O’Rourke B (2018) Mitochondrial ROS drive sudden cardiac death and chronic proteome remodeling in heart failure. Circ Res 123(3):356-371. https:// doi.org/10.1161/CIRCRESAHA.118.312708

40. Schiattarella GG, Boccella N, Paolillo R et al (2018) Loss of akap1 exacerbates pressure Overload-Induced cardiac hypertrophy and heart failure. Front Physiol 9:558. https://doi.org/10.3389/fphys. 2018.00558

Publisher's note Springer Nature remains neutral with regard to jurisdictional claims in published maps and institutional affiliations. 